\title{
Multiple-Description Vector Quantization with Lattice Codebooks: Design and Analysis
}

\author{
Vinay A. Vaishampayan, Member, IEEE, N. J. A. Sloane, Fellow, IEEE, and Sergio D. Servetto, Member, IEEE
}

\begin{abstract}
The problem of designing a multiple-description vector quantizer with lattice codebook $\Lambda$ is considered. A general solution is given to a labeling problem which plays a crucial role in the design of such quantizers. Numerical performance results are obtained for quantizers based on the lattices $A_{2}$ and $\mathbb{Z}^{i}$, $i=1,2,4,8$, that make use of this labeling algorithm.

The high-rate squared-error distortions for this family of $L$-dimensional vector quantizers are then analyzed for a memoryless source with probability density function (pdf) $p$ and differential entropy $h(p)<\infty$. For any $a \in(0,1)$ and rate pair $(R, R)$, it is shown that the two-channel distortion $\bar{d}_{0}$ and the channel 1 (or channel 2) distortion $\bar{d}_{s}$ satisfy
\end{abstract}

and

$$
\lim _{R \rightarrow \infty} \bar{d}_{0} 2^{2 R(1+a)}=\frac{1}{4} G(\Lambda) 2^{2 h(p)}
$$

$$
\lim _{R \rightarrow \infty} \bar{d}_{s} 2^{2 R(1-a)}=G\left(S_{L}\right) 2^{2 h(p)}
$$

where $G(\Lambda)$ is the normalized second moment of a Voronoi cell of the lattice $\Lambda$ and $G\left(S_{L}\right)$ is the normalized second moment of a sphere in $L$ dimensions.

Index Terms-Cubic lattice, hexagonal lattice, lattice quantization, multiple descriptions, quantization, source coding, vector quantization.

\section{INTRODUCTION}

W E consider the problem of designing a multiple-description vector quantizer for a memoryless source with probability density function (pdf) $p$, differential entropy $h(p)<\infty$, and the squared-error distortion measure. A multiple-description vector quantizer encodes vectors for transmission over a two-channel communication system. The objective is to send information about the source vector over each channel in such a way that good performance is obtained when both channels work and the degradation is small when either channel fails. It is assumed that the encoder has no knowledge about the state of a channel, i.e., it does not know whether a channel has failed or is working.

The recent interest in the multiple-description problem is largely due to the application to image, video, and voice communications over packet networks with nonzero probability of packet loss. The loss of a packet could be significant if it results

Manuscript received January 26, 2000; revised October 7, 2000.

V. A. Vaishampayan and N. J. A. Sloane are with AT\&T Shannon Laboratory, Florham Park, NJ 07932 USA (e-mail: vinay@ research.att.com; njas@ research.att.com).

S. D. Servetto is with Ecole Polytechnique Fédérale de Lausanne, CH-1015 Lausanne, Switzerland (e-mail: sevetto@lcavsunl.epfl.ch).

Communicated by P. A. Chou, Associate Editor for Source Coding.

Publisher Item Identifier S 0018-9448(01)04412-1. in a loss of a large block of source samples, such as a large image block or a large block of speech. One way to improve performance is to place different encoded versions of a given block of source samples into several packets in such a way that if some of these packets are received, a degraded version of the source block may be recovered. This leads to the formulation of the multiple-description source coding problem.

For the single-description problem, one of the benefits of vector quantization over scalar quantization is a reduction in granular distortion. This is because in higher dimensions it is possible to construct Voronoi cells that are more "spherical" than the hypercube. To be more specific, uniform scalar quantization coupled with entropy coding is known to have mean squared error (mse) $\bar{d}(R)$ at entropy $R$ bits per sample satisfying [16]

$$
\lim _{R \rightarrow \infty} \bar{d}(R) 2^{2 R}=\frac{2^{2 h(p)}}{12}
$$

whereas if an $L$-dimensional lattice $\Lambda$ is used as a codebook, the distortion satisfies

$$
\lim _{R \rightarrow \infty} \bar{d}(R) 2^{2 R}=G(\Lambda) 2^{2 h(p)}
$$

where $G(\Lambda)$ is the normalized second moment of a Voronoi cell of the lattice. In dimensions greater than one, lattices exist for which $G(\Lambda)$ is strictly smaller than $1 / 12$. For example, in eight dimensions, it is possible to gain $0.66 \mathrm{~dB}$ by using the lattice $E_{8}$ as compared to uniform scalar quantization [11]. It is also known through a random quantizing argument [31] that quantizers exist for which the product $\bar{d}(R) 2^{2 R}$ approaches $2^{2 h(p)} /(2 \pi e)$ as the rate increases. Furthermore, it follows from rate distortion theory [5] that no smaller value can be achieved for the above product in the limit of infinite rate. The maximum gain possible over entropy-coded scalar quantization is $1.53 \mathrm{~dB}$ and lattices provide a useful method for closing this gap.

From now on we will restrict our attention to the case of two channels. Consider a multiple-description quantizer which sends information across each channel at a rate of $R$ bits per sample. The performance of this system is measured in terms of three distortions: the two-channel distortion $\bar{d}_{0}$, when both descriptions are available to the decoder; the channel 1 distortion $\bar{d}_{1}$, when only the first description is available; and the channel 2 distortion $\bar{d}_{2}$, when only the second description is available. We will further assume that $\bar{d}_{1}=\bar{d}_{2}=\bar{d}_{s}$ and will refer to this common value as the side distortion. The objective is to design vector quantizers that minimize $\bar{d}_{0}$ under the constraint $\bar{d}_{s} \leq D_{s}$, for a given rate pair $(R, R)$ and a given bound $D_{s}$ on the side-channel distortion. 


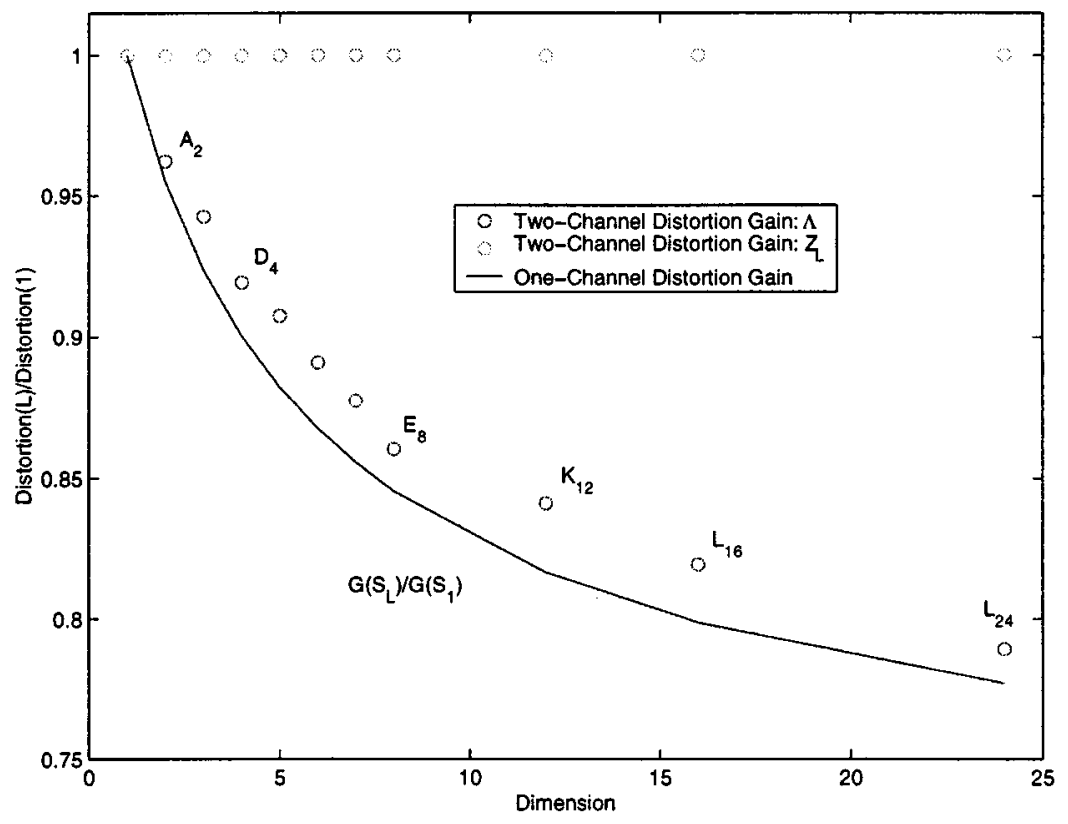

Fig. 1. Limiting two-channel and single-channel distortion ratios $\lim _{R \rightarrow \infty} \bar{d}_{0}(R, L) / \bar{d}_{0}(R, 1)$ and $\lim _{R \rightarrow \infty} \bar{d}_{s}(R, L) / \bar{d}_{1}(R, 1)$ as a function of the lattice dimension $L$. The reduction in two-channel distortion is lattice dependent and is determined by the normalized second moment of a Voronoi region of the lattice. The reduction in single-channel distortion depends only on the dimension of the lattice.

It has been shown [27] that for a uniform entropy-coded multiple-description quantizer, and any $a \in(0,1)$, the distortions satisfy

$$
\begin{aligned}
& \lim _{R \rightarrow \infty} \bar{d}_{0}(R) 2^{2 R(1+a)}=\frac{1}{4}\left(\frac{2^{2 h(p)}}{12}\right) \\
& \lim _{R \rightarrow \infty} \bar{d}_{s}(R) 2^{2 R(1-a)}=\left(\frac{2^{2 h(p)}}{12}\right) .
\end{aligned}
$$

On the other hand, by using a random quantizer argument it was shown [28] that by encoding vectors of infinite block length, it is possible to achieve distortions

$$
\begin{aligned}
& \lim _{R \rightarrow \infty} \bar{d}_{0}(R) 2^{2 R(1+a)}=\frac{1}{4}\left(\frac{2^{2 h(p)}}{2 \pi e}\right) \\
& \lim _{R \rightarrow \infty} \bar{d}_{s}(R) 2^{2 R(1-a)}=\left(\frac{2^{2 h(p)}}{2 \pi e}\right) .
\end{aligned}
$$

Thus, by using multiple-description quantization, it is possible to simultaneously reduce the two-channel and side-channel granular distortions by $1.53 \mathrm{~dB}$.

In single-description quantization, an extra transmitted bit reduces the squared error distortion by a factor of 4 (this is seen in (1)). However, in multiple-description quantization there is additional flexibility. If each $R$ is increased by $1 / 2$ bit, the two-channel distortion can be decreased by $2^{-(1+a)}$ and the side distortion by $2^{-(1-a)}$, for any $a \in(0,1)$. This means that by using an extra bit, the distortions $\bar{d}_{0}$ and $\bar{d}_{s}$ can be made to decrease by different amounts as long as the product decreases by a factor of 4 .

The goal of this paper is to give constructions for closing this "1.53-dB" gap and to analyze the resulting performance gains. Our approach is as follows. From classical quantization theory, we know that the gap between scalar quantization and the rate- distortion bound may be closed by using vector quantizers with lattice codebooks. Certainly, by following this approach we can also close the gap between the two-channel distortion and the rate-distortion bound. In particular, this will allow us to replace the factor $(1 / 12)$ in the expression for $\bar{d}_{0}$ in (3) with $G(\Lambda)$, the normalized second moment of the Voronoi region of a lattice point. The main question we address here is that of simultaneously reducing $\bar{d}_{s}$. How can such a reduction be achieved and what is the quantity that will replace the factor $(1 / 12)$ in the expression for $\bar{d}_{s}$ in (3)? We will show through a constructive procedure that the distortion $\bar{d}_{s}$ can be reduced by solving a specific labeling problem. To our surprise, the quantity that replaces $(1 / 12)$ is $G\left(S_{L}\right)$, the normalized second moment of a sphere in $L$ dimensions.

Let $\bar{d}_{0}(R, L)$ and $\bar{d}_{s}(R, L)$ denote the two-channel and single-channel distortions at rate $R$ for an $L$-dimensional quantizer. Fig. 1 summarizes the main results of the paper. In this figure, we have plotted the limit of the normalized two-channel and single-channel distortions $\lim _{R \rightarrow \infty} \bar{d}_{0}(R, L) / \bar{d}_{0}(R, 1)$ and $\lim _{R \rightarrow \infty} \bar{d}_{s}(R, L) / \bar{d}_{s}(R, 1)$, respectively, for lattices of various dimensions. It is seen that the limit for the two-channel distortion is given by the ratio $G(\Lambda) / G(\mathbb{Z})$, which depends on the lattice, whereas for the side distortion the limit is $G\left(S_{L}\right) / G\left(S_{1}\right)$, which is independent of the lattice.

\section{Previous Work}

An achievable rate region for the multiple-description problem was first given in [12] and it was shown in [24] that this region coincides with the rate-distortion region for a memoryless Gaussian source with a squared-error distortion measure. The problem of multiple-description quantizer design, including a formulation and solution of the underlying labeling problem in one dimension, was presented in [26]. An asymptotic performance analysis of this quantizer was 


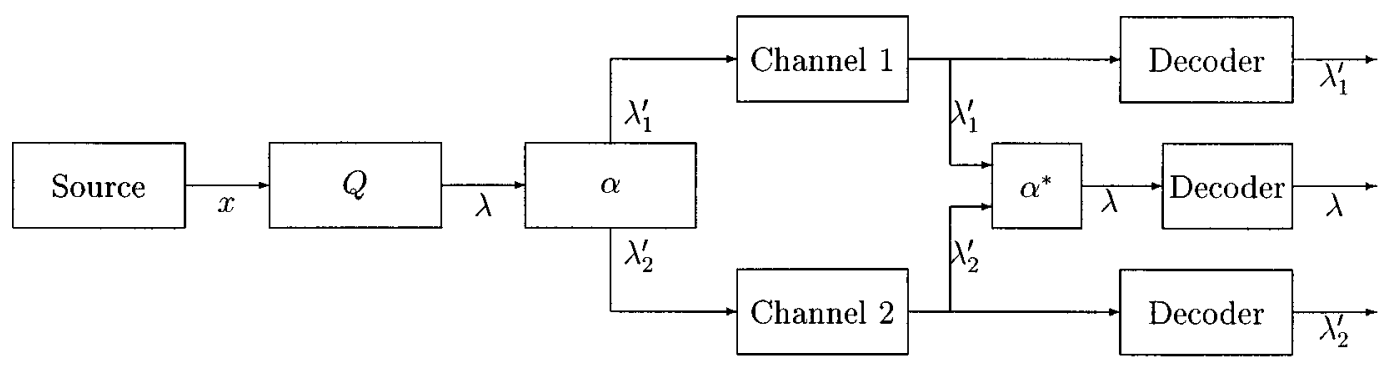

Fig. 2. Block diagram for a MDVQ.

presented in [27]. A preliminary version of the work presented here was first presented in [25].

Lattice quantizers (for the single-description problem) have been extensively studied. In [31], a random-quantization argument is used to give upper and lower bounds on the performance of quantizers for a fixed dimension. Detailed descriptions of the Voronoi regions of specific lattices are given in [9] and their second moments are evaluated. Fast quantizing algorithms for lattice quantizers are given in [10], [1], [14], and [30]. In [15], it is conjectured that for $r$ th-power difference distortion measures, any optimal quantizer, in any dimension, has Voronoi regions that are congruent to some polytope.

Approaches to multiple-description coding based on trelliscoded quantization are presented in [28], [20]; those based on vector quantization are presented in [13]; and approaches using forward error correction are presented in [22].

There are also approaches to multiple-description coding based on subspace methods. One approach is to design a predictor or a transform so as to achieve a correlation structure that allows one half of the prediction error samples or transform coefficients to be predicted from the other half. Examples of this approach are presented in [19], [23], and [29].

Another approach to multiple-description coding is based on using overcomplete expansions. Here, the idea is to construct a redundant signal representation, in a way that allows the signal to be estimated with a controlled amount of error when the representation is incompletely received [3], [7], [17].

\section{ORGANIZATION OF THE PAPER}

The multiple-description vector quantizer (MDVQ) is described, notation is established, certain regularity assumptions for the labeling function are stated, and preliminary expressions are derived for rates and distortions in Section IV. A detailed example with the two-dimensional hexagonal lattice $A_{2}$ is presented along with some general theory in Section V. The necessary theory for general lattices is presented in Section VI. An asymptotic analysis for a fixed vector dimension is presented in Section VII. The paper is summarized along with conclusions and directions for future work in Section VIII.

\section{PRELIMINARIES}

A block diagram of an MDVQ with a lattice codebook is shown in Fig. 2.
A source of information generates a sequence of independent and identically distributed random variables with pdf $p$. This source is blocked off into $L$-dimensional vectors $x=\left(x_{1}, x_{2}, \ldots, x_{L}\right)$. The $L$-fold pdf is denoted by $p_{L}$, where

$$
p_{L}(x)=\prod_{i=1}^{L} p\left(x_{i}\right)
$$

The vector $x$ is quantized to the nearest vector $\lambda$ in a lattice $\Lambda \subset \mathbb{R}^{L}$. We denote the quantizer mapping by $\lambda=Q(x)$. Information about the selected code vector $\lambda$ is then sent across the two channels, subject to rate constraints imposed by the individual channels. This is done through a labeling function $\alpha$ followed by entropy coding. The labeling function $\alpha$ maps $\lambda \in \Lambda$ to a pair $\left(\lambda_{1}^{\prime}, \lambda_{2}^{\prime}\right) \in \Lambda^{\prime} \times \Lambda^{\prime}$, where $\Lambda^{\prime}$ is a sublattice of $\Lambda$ with index $N$. The component functions of $\alpha$ are denoted by $\alpha_{1}$ and $\alpha_{2}$, where $\alpha_{1}(\lambda)=\lambda_{1}^{\prime}$ and $\alpha_{2}(\lambda)=\lambda_{2}^{\prime}$. For simplicity, we assume that $\Lambda^{\prime}$ is geometrically similar to $\Lambda$, i.e., $\Lambda^{\prime}$ can be obtained by scaling, rotating, and possibly reflecting $\Lambda$. Note that points in the lattice $\Lambda$ are denoted by $\lambda$, possibly with subscripts, whereas sublattice points will be denoted by $\lambda^{\prime}$ or $\lambda^{\prime \prime}$, possibly with subscripts.

In Fig. 3, a portion of the hexagonal lattice $A_{2}$ is illustrated, along with a geometrically similar sublattice of index 31 . The lattice points lie at the intersection of the straight lines in the hexagonal grid (only some of the points are shown). The sublattice points are marked with upper case letters. Observe that the lattice is 31 times as dense as the sublattice, i.e., there are 31 lattice points for every sublattice point.

At the decoder, if only channel 1 works, the received information is used to decode $\lambda_{1}^{\prime}$, and if only channel 2 works, the information received over channel 2 is used to decode $\lambda_{2}^{\prime}$. The mapping $\alpha$ is assumed to be one-to-one so that if both channels work, $\lambda$ can be recovered from $\left(\lambda_{1}^{\prime}, \lambda_{2}^{\prime}\right)$. (In practice, if only one channel is working it may be better to decode the received vector to some function of $\lambda_{1}^{\prime}$ or $\lambda_{2}^{\prime}$ rather than to $\lambda_{1}^{\prime}$ or $\lambda_{2}^{\prime}$ itself. If $\lambda_{1}^{\prime}$ is received but $\lambda_{2}^{\prime}$ is not, for instance, we would decode $\lambda_{1}^{\prime}$ as the center of mass of all points $\lambda \in \Lambda$ such that the first component of $\alpha(\lambda)$ is $\lambda_{1}^{\prime}$. We will ignore this complication in order to simplify the analysis.)

Given $\Lambda, \Lambda^{\prime}$, and $\alpha$, there are three distortions and two rates associated with an MDVQ. For a given $x$ mapped to the triple $\left(\lambda, \lambda_{1}^{\prime}, \lambda_{2}^{\prime}\right)$ by the MDVQ, the two-channel distortion $d_{0}$ is given by $\|x-\lambda\|^{2}$, the channel 1 distortion $d_{1}$ by $\left\|x-\lambda_{1}^{\prime}\right\|^{2}$, and the channel 2 distortion $d_{2}$ by $\left\|x-\lambda_{2}^{\prime}\right\|^{2}$ (we assume that the 


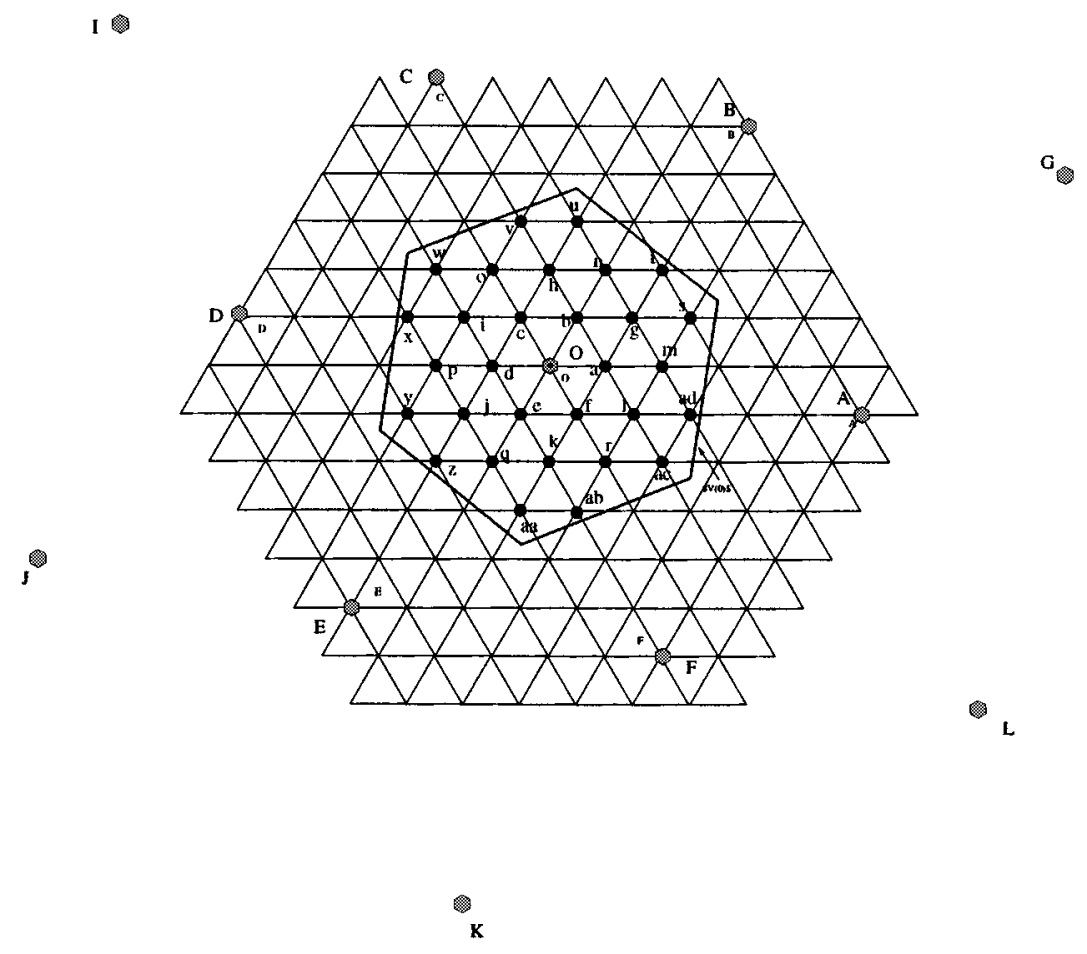

Fig. 3. This figure shows a portion of the $A_{2}$ lattice (the points on the grid line intersections), part of a geometrically similar sublattice of index 31 , a discrete Voronoi set for a sublattice point (the lattice points in the hexagon) and the Voronoi set of a sublattice point (all the points in the hexagon).

inner product of $L$-dimensional vectors $x=\left(x_{1}, x_{2}, \ldots, x_{L}\right)$ and $y=\left(y_{1}, y_{2}, \ldots, y_{L}\right)$ is given by

$$
\langle x, y\rangle=\frac{1}{L} \sum_{i=1}^{L} x_{i} y_{i}
$$

and the corresponding norm is $\|x\|=\langle x, x\rangle^{1 / 2}$, i.e., the inner product and norm are dimension-normalized). The corresponding average distortions are denoted by $\bar{d}_{0}, \bar{d}_{1}$, and $\bar{d}_{2}$. We assume that an entropy coder is used in order to transmit the labeled vectors at a rate arbitrarily close to the entropy, i.e.,

$$
R_{i}=\mathcal{H}\left(\alpha_{i}(Q(\boldsymbol{X}))\right) / L, \quad i=1,2
$$

where $\mathcal{H}(U)$, the entropy of the random variable $U$ taking values in alphabet $\mathcal{U}$ with probability distribution $P$, is given by

$$
\mathcal{H}(U)=-\sum_{u \in \mathcal{U}} P(u) \log P(u)
$$

The problem is to design the labeling function $\alpha$ so as to minimize $\bar{d}_{0}$ subject to $\bar{d}_{1} \leq D_{s}, \bar{d}_{2} \leq D_{s}$, and $R_{i} \leq R, i=1,2$, for specified values of the rate $R$ and distortion $\bar{D}_{s}$.

The Voronoi (or nearest neighbor) region $V(\lambda)$ of a point $\lambda \in \Lambda$ is defined to be

$$
V(\lambda) \stackrel{\text { def }}{=}\{x:\|x-\lambda\| \leq\|x-\tilde{\lambda}\|, \forall \tilde{\lambda} \in \Lambda\} .
$$

With each sublattice point $\lambda^{\prime} \in \Lambda^{\prime}$ we associate a discrete Voronoi set (with $N$ elements)

$$
V_{0}\left(\lambda^{\prime}\right) \stackrel{\text { def }}{=}\left\{\lambda \in \Lambda:\left\|\lambda-\lambda^{\prime}\right\| \leq\left\|\lambda-\lambda^{\prime \prime}\right\|, \forall \lambda^{\prime \prime} \in \Lambda^{\prime}\right\} .
$$

In (5) and (6), ties (i.e., points for which equality holds in the defining condition) are broken in some prearranged manner. The existence of sublattices for which no ties occur is discussed in [8]. The Voronoi region $V(0)$ and the discrete Voronoi region $V_{0}(0)$ are both illustrated in Fig. 3.

We regard the label for $\lambda$ as a directed edge $\vec{e}=$ $\left(\alpha_{1}(\lambda), \alpha_{2}(\lambda)\right)$ of the graph with vertex set $\Lambda^{\prime}$. The corresponding unordered pair $e=\left\{\alpha_{1}(\lambda), \alpha_{2}(\lambda)\right\}$ will be referred to as the undirected edge or undirected label associated with $\lambda$. The essential difference between a directed edge or label $\left(\lambda_{a}^{\prime}, \lambda_{b}^{\prime}\right)$ and the undirected edge $\left\{\lambda_{a}^{\prime}, \lambda_{b}^{\prime}\right\}$ is that for the directed edge there is an implicit association between edge component and channel ( $\lambda_{a}^{\prime}$ is sent on channel 1 and $\lambda_{b}^{\prime}$ is sent on channel 2) whereas for the undirected edge no association is implied. Graphically, an edge connecting two sublattice points $\lambda_{a}^{\prime}$ and $\lambda_{b}^{\prime}$, with an arrow pointing from $\lambda_{a}^{\prime}$ to $\lambda_{b}^{\prime}$, indicates that $\lambda_{a}^{\prime}$ is sent on channel 1 and $\lambda_{b}^{\prime}$ is sent on channel 2, or, equivalently, the directed edge is $\left(\lambda_{a}^{\prime}, \lambda_{b}^{\prime}\right)$. The two directed versions of an (undirected) edge $e$ will be denoted $\vec{e}$ and $\overleftarrow{e}$.

For a given labeling function $\alpha$, an associated undirected edge labeling function $\alpha_{u}$ is defined as follows: if $\alpha(\lambda)=\vec{e}$, then $\alpha_{u}(\lambda)=e$, i.e., $\alpha_{u}$ maps $\lambda$ to its undirected label. Note that 

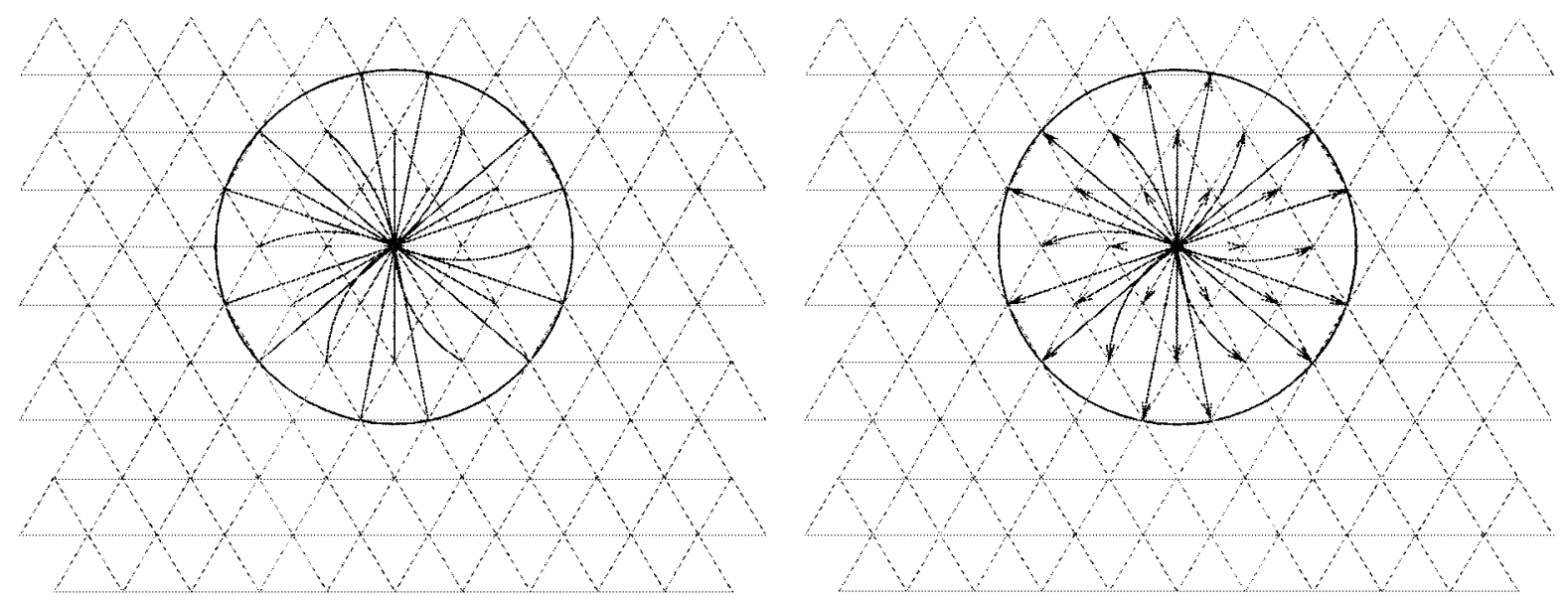

Fig. 4. Various edge sets are illustrated for a sublattice of $A_{2}$ with $N=31$. For clarity, only the sublattice points are shown. The set $\mathcal{E}_{u \mid \alpha_{1}=0}$ is shown on the left, $\mathcal{E}_{d \mid \alpha_{1}=0}$ on the right. The origin is located at the center of the circle.

if $\alpha\left(\lambda_{1}\right)=\vec{e}$ and $\alpha\left(\lambda_{2}\right)=\overleftarrow{e}$, then $\alpha_{u}\left(\lambda_{1}\right)=\alpha_{u}\left(\lambda_{2}\right)=e$, i.e., $\alpha_{u}$ is not one-to-one. A directed edge is uniquely associated with a lattice point whereas an undirected edge will in general be associated with two lattice points, one for each orientation of the edge. The main reason for introducing $\alpha_{u}$ is that its construction logically precedes that of $\alpha$.

Certain sets associated with the two maps will play a central role in the development. The first is $\mathcal{E}_{d}\left(\lambda^{\prime}\right)=\alpha\left(V_{0}\left(\lambda^{\prime}\right)\right)$, the set of all labels for points in $V_{0}\left(\lambda^{\prime}\right)$ (the subscript $d$ indicates that the edges are directed). The corresponding set of undirected edges is denoted by $\mathcal{E}_{u}\left(\lambda^{\prime}\right)$. More specifically, $\mathcal{E}_{u}\left(\lambda^{\prime}\right)=$ $\alpha_{u}\left(V_{0}\left(\lambda^{\prime}\right)\right)$. The set of all (directed) labels is denoted by $\mathcal{E}_{\boldsymbol{d}}=$ $\bigcup_{\lambda^{\prime} \in N^{\prime}} \mathcal{E}_{d}\left(\lambda^{\prime}\right)$ and the set of all undirected labels by $\mathcal{E}_{u}=$ $\bigcup_{\lambda^{\prime} \in \Lambda^{\prime}} \mathcal{E}_{u}\left(\lambda^{\prime}\right)$. It is also useful to define the restriction

$$
\left.\mathcal{E}_{d}\right|_{\alpha_{1}=0}=\left\{\left(\lambda_{1}^{\prime}, \lambda_{2}^{\prime}\right) \in \mathcal{E}_{d}, \lambda_{1}^{\prime}=0\right\} .
$$

The set of undirected edges in $\left.\mathcal{E}_{d}\right|_{\alpha_{1}=0}$ will be denoted by $\left.\mathcal{E}_{u}\right|_{\alpha_{1}=0}$. The sets $\left.\mathcal{E}_{u}\right|_{\alpha_{1}=0}$ and $\left.\mathcal{E}_{d}\right|_{\alpha_{1}=0}$ are illustrated in Fig. 4 .

The reuse index associated with a label $\lambda^{\prime}$ and channel $i$ is defined to be $N_{i}\left(\lambda^{\prime}\right)=\left|\left\{\lambda: \alpha_{i}(\lambda)=\lambda^{\prime}\right\}\right|$, the number of lattice points $\lambda$ for which $\alpha_{i}(\lambda)=\lambda^{\prime}$.

In order to render the problem tractable, we assume that the labeling function has the following properties.

- Property 1: The reuse index $N_{i}\left(\lambda^{\prime}\right)=N$, for all $\lambda^{\prime} \in \Lambda^{\prime}$ and $i=1,2$. In other words, each channel $i$ label is reused exactly $N$ times.

- Property 2: The shift property: $\alpha_{u}\left(\lambda+\lambda^{\prime}\right)=\alpha_{u}(\lambda)+\lambda^{\prime}$, for all $\lambda \in \Lambda$ and $\lambda^{\prime} \in \Lambda^{\prime}$.

- Property 3: Each undirected edge $e=\left\{\lambda_{1}^{\prime}, \lambda_{2}^{\prime}\right\}, \lambda_{1}^{\prime} \neq$ $\lambda_{2}^{\prime}$, labels two points, $\lambda_{a}$ and $\lambda_{b}$, and $\lambda_{a}+\lambda_{b}=\lambda_{1}^{\prime}+\lambda_{2}^{\prime}$.

The first assumption makes it easy to parameterize the tradeoff between the side and central distortions. The second assumption reduces the labeling problem to one of labeling a finite set. The third assumption is a simple way to achieve exact balance between the two descriptions (its implications will become clearer in Section V-B3).

It will prove useful to have the following definitions of equivalence.
Definition 1: Two lattice points $\lambda_{1}$ and $\lambda_{2}$ are said to be equivalent if either $\lambda_{1}$ and $\lambda_{2}$ or $\lambda_{1}$ and $-\lambda_{2}$ lie in the same coset of $\Lambda$ relative to the sublattice $\Lambda^{\prime}$.

Definition 2: Two sublattice edges $e_{1}$ and $e_{2}$ are said to be equivalent if they are parallel and of equal length, or equivalently if $e_{1}+\lambda^{\prime}=e_{2}$ for some $\lambda^{\prime} \in \Lambda^{\prime}$.

The equivalence class of an object will be indicated by square brackets. Thus, $[\lambda]$ is the equivalence class of $\lambda$ and $[e]$ is the equivalence class of $e$, or, equivalently, $[\lambda] \in \Lambda / \Lambda^{\prime}$ and $[e] \in$ $\mathcal{E}_{u} / \Lambda^{\prime}$.

\section{A. Distortion Computation}

The average two-channel distortion $\bar{d}_{0}$ is given by

$$
\bar{d}_{0}=\sum_{\lambda \in \Lambda} \int_{V(\lambda)}\|x-\lambda\|^{2} p_{L}(x) d x .
$$

Since the codebook of the quantizer is a lattice, all the Voronoi regions are congruent. Furthermore, upon assuming that each Voronoi region is small so that $p_{L}(x) \approx p_{L}(\lambda)$ for $x \in V(\lambda)$ and upon letting $\nu$ denote the $L$-dimensional volume of a Voronoi region, we obtain the following expression for the two-channel distortion [15]:

$$
\bar{d}_{0} \approx \frac{\int_{V(0)}\|x\|^{2} d x}{\nu}
$$

which in terms of the normalized second moment $G(\Lambda)$, defined by

$$
G(\Lambda) \stackrel{\text { def }}{=} \frac{\int_{V(0)}\|x\|^{2} d x}{\nu^{1+2 / L}}
$$

is given by

$$
\bar{d}_{0} \approx G(\Lambda) \nu^{2 / L} .
$$

We now derive expressions for the average distortions $\bar{d}_{1}$ and $\bar{d}_{2}$ and

$$
\bar{d}_{s} \stackrel{\text { def }}{=}\left(\bar{d}_{1}+\bar{d}_{2}\right) / 2 .
$$


When only description 1 is available, the distortion is given by

$$
\begin{aligned}
\bar{d}_{1}= & \sum_{\lambda \in \Lambda} \int_{V(\lambda)}\left\|x-\alpha_{1}(\lambda)\right\|^{2} p_{L}(x) d x \\
= & \sum_{\lambda \in \Lambda} \int_{V(\lambda)}\left\|x-\lambda+\lambda-\alpha_{1}(\lambda)\right\|^{2} p_{L}(x) d x \\
= & \sum_{\lambda \in \Lambda} \int_{V(\lambda)}\|x-\lambda\|^{2} p_{L}(x) d x \\
& +\sum_{\lambda \in \Lambda} \int_{V(\lambda)}\left\|\lambda-\alpha_{1}(\lambda)\right\|^{2} p_{L}(x) d x \\
& +2 \sum_{\lambda \in \Lambda} \int_{V(\lambda)}\left\langle x-\lambda, \lambda-\alpha_{1}(\lambda)\right\rangle p_{L}(x) d x \\
= & \bar{d}_{0}+\sum_{\lambda \in \Lambda}\left\|\lambda-\alpha_{1}(\lambda)\right\|^{2} P(\lambda)+2 \sum_{\lambda \in \Lambda} \\
& \cdot\left\langle\int_{V(\lambda)} x p_{L}(x) d x-\int_{V(\lambda)} \lambda p_{L}(x) d x, \lambda-\alpha_{1}(\lambda)\right\rangle \\
\stackrel{(a)}{=} & \bar{d}_{0}+\sum_{\lambda \in \Lambda}\left\|\lambda-\alpha_{1}(\lambda)\right\|^{2} P(\lambda)
\end{aligned}
$$

where $P(\lambda)=\operatorname{Pr}(Q(X)=\lambda)$, and (a) follows by assuming that $\lambda$ is the centroid of its Voronoi region. This is true for the uniform density. For nonuniform densities, there is an error term which goes to zero with the size of the Voronoi region. The first term in (12) is the two-channel distortion and the second term is the excess distortion which is incurred when channel 2 fails. Note that for a given $\Lambda$, only the excess distortion term is affected by the labeling function $\alpha$. From (12), it follows that

$$
\begin{aligned}
\bar{d}_{s}= & \bar{d}_{0}+(1 / 2) \sum_{\lambda \in \Lambda}\left(\left\|\lambda-\alpha_{1}(\lambda)\right\|^{2}+\left\|\lambda-\alpha_{2}(\lambda)\right\|^{2}\right) P(\lambda) \\
= & \bar{d}_{0}+(1 / 2) \sum_{\lambda^{\prime} \in \Lambda^{\prime}} \sum_{\lambda \in V_{0}\left(\lambda^{\prime}\right)} \\
& \cdot\left(\left\|\lambda-\alpha_{1}(\lambda)\right\|^{2}+\left\|\lambda-\alpha_{2}(\lambda)\right\|^{2}\right) P(\lambda) .
\end{aligned}
$$

We also introduce the following notation:

$$
d_{1}(\lambda, \vec{e})=\left\|\lambda-\lambda_{1}^{\prime}\right\|^{2} \quad \text { and } \quad d_{2}(\lambda, \vec{e})=\left\|\lambda-\lambda_{2}^{\prime}\right\|^{2}
$$

and

$$
d_{s}(\lambda, \vec{e})=\left(d_{1}(\vec{e})+d_{2}(\vec{e})\right) / 2, \quad \text { where } \vec{e}=\left(\lambda_{1}^{\prime}, \lambda_{2}^{\prime}\right) \text {. }
$$

Note that $d_{s}(\lambda, \vec{e})=d_{s}(\lambda, \overleftarrow{e})$. Hence, when appropriate, we will write $d_{s}(\lambda, e)$. Also, when the edge associated with the lattice point is clear from the context, we will write $d_{s}(\lambda)$ or $d_{s}(\vec{e})$ instead of $d_{s}(\lambda, \vec{e})$. It is useful (as a design guide and for the asymptotics which follow) to write down a slightly different expression for the side distortion where the sum is taken over the edge set $\mathcal{E}_{d}=\bigcup_{\lambda^{\prime} \in \Lambda^{\prime}} \mathcal{E}_{d}\left(\lambda^{\prime}\right)$

$$
\begin{aligned}
\bar{d}_{s} & =\bar{d}_{0}+\sum_{\vec{e} \in \mathcal{E}_{d}} d_{s}(\vec{e}) P(\vec{e}) \\
& =\bar{d}_{0}+\sum_{\lambda^{\prime} \in \Lambda^{\prime}} \sum_{\vec{e} \in \mathcal{E}_{d}\left(\lambda^{\prime}\right)} d_{s}(\vec{e}) P(\vec{e})
\end{aligned}
$$

where $P(\vec{e})$ is equal to the probability of the lattice point that the edge labels, i.e., $P(\vec{e})=\operatorname{Pr}(Q(X)=\lambda)$.

\section{B. Rate Computation}

Expressions for the rate (in bits per sample) will be derived next. Let $R_{0}$ be the rate required to address the two-channel codebook for a single-channel system. ${ }^{1}$ We will first derive an expression for $R_{0}$ and then determine the (per-channel) rate $R$ of the multiple-description system.

In order to derive expressions for $R_{0}$ and $R$, we use the fact that each quantizer bin has identical volume $\nu$ and that $p_{L}(x)$ is approximately constant over Voronoi regions of the sublattice $V\left(\lambda^{\prime}\right)$. The second assumption is valid in the limit as the Voronoi regions become small and is standard in asymptotic quantization theory.

The rate $R_{0}=\mathcal{H}(Q(X))$ is given by [18]

$$
\begin{aligned}
R_{0} & =-(1 / L) \sum_{\lambda} \int_{V(\lambda)} p_{L}(x) d x \log _{2} \int_{V(\lambda)} p_{L}(x) d x \\
& \approx-(1 / L) \sum_{\lambda} \int_{V(\lambda)} p_{L}(x) d x \log _{2} p_{L}(\lambda) \nu \\
& \approx h(p)-(1 / L) \log _{2}(\nu) .
\end{aligned}
$$

For $R$, we evaluate the entropy $\mathcal{H}\left(\alpha_{1}(Q(X))\right)$ and then use the approximation that $p_{L}(x)$ is roughly constant over each Voronoi region of $\Lambda^{\prime}$ to get

$$
\begin{aligned}
R= & -(1 / L) \sum_{\lambda^{\prime} \in \Lambda^{\prime}}\left(\sum_{\lambda \in \alpha_{1}^{-1}\left(\lambda^{\prime}\right)} \int_{V(\lambda)} p_{L}(x) d x\right) \\
& \cdot \log _{2}\left[\left(\sum_{\lambda \in \alpha_{1}^{-1}\left(\lambda^{\prime}\right)} \int_{V(\lambda)} p_{L}(x) d x\right)\right] \\
\approx & -(1 / L) \sum_{\lambda^{\prime} \in \Lambda^{\prime}}\left(\sum_{\lambda \in \alpha_{1}^{-1}\left(\lambda^{\prime}\right)} \int_{V(\lambda)} p_{L}(x) d x\right) \\
& \cdot \log _{2}\left(p_{L}\left(\lambda^{\prime}\right) N \nu\right) \\
\approx & -(1 / L) \sum_{\lambda^{\prime} \in \Lambda^{\prime}}\left(\sum_{\lambda \in \alpha_{1}^{-1}\left(\lambda^{\prime}\right)} \int_{V(\lambda)} p_{L}(x) \log _{2}\left(p_{L}\left(\lambda^{\prime}\right)\right) d x\right) \\
& -(1 / L) \log _{2}(N \nu) \\
\approx & h(p)-(1 / L) \log _{2}(N \nu) .
\end{aligned}
$$

Observe that in the above equation, the term $N \nu$ is simply the volume of a fundamental region for the sublattice $\Lambda^{\prime}$ (since it has index $N$ in $\Lambda$ ). Upon writing (17) in terms of $R_{0}$ we obtain

$$
R=R_{0}-(1 / L) \log _{2}(N) \text {. }
$$

A single-channel system would have used $R_{0}$ bits per sample to achieve the same $\bar{d}_{0}$. Instead, a multiple-description system uses a total of $2 R=2 R_{0}-(2 / L) \log _{2}(N)$ bits per sample, and so the rate overhead is $R_{0}-(2 / L) \log _{2}(N)$.

\section{A LABELING FunCTION FOR $A_{2}$}

We now look for a labeling function $\alpha$ for which

$$
\sum_{\vec{e} \in \mathcal{E}_{d}\left(\lambda^{\prime}\right)} d_{s}(\vec{e})
$$

is minimized and is independent of $\lambda^{\prime}$. Since the details are complicated, we will work out the first example—for the hexagonal

\footnotetext{
${ }^{1}$ This quantity is useful for evaluating the two-channel distortion as well as for
} evaluating the rate overhead associated with the multiple-description scheme. 


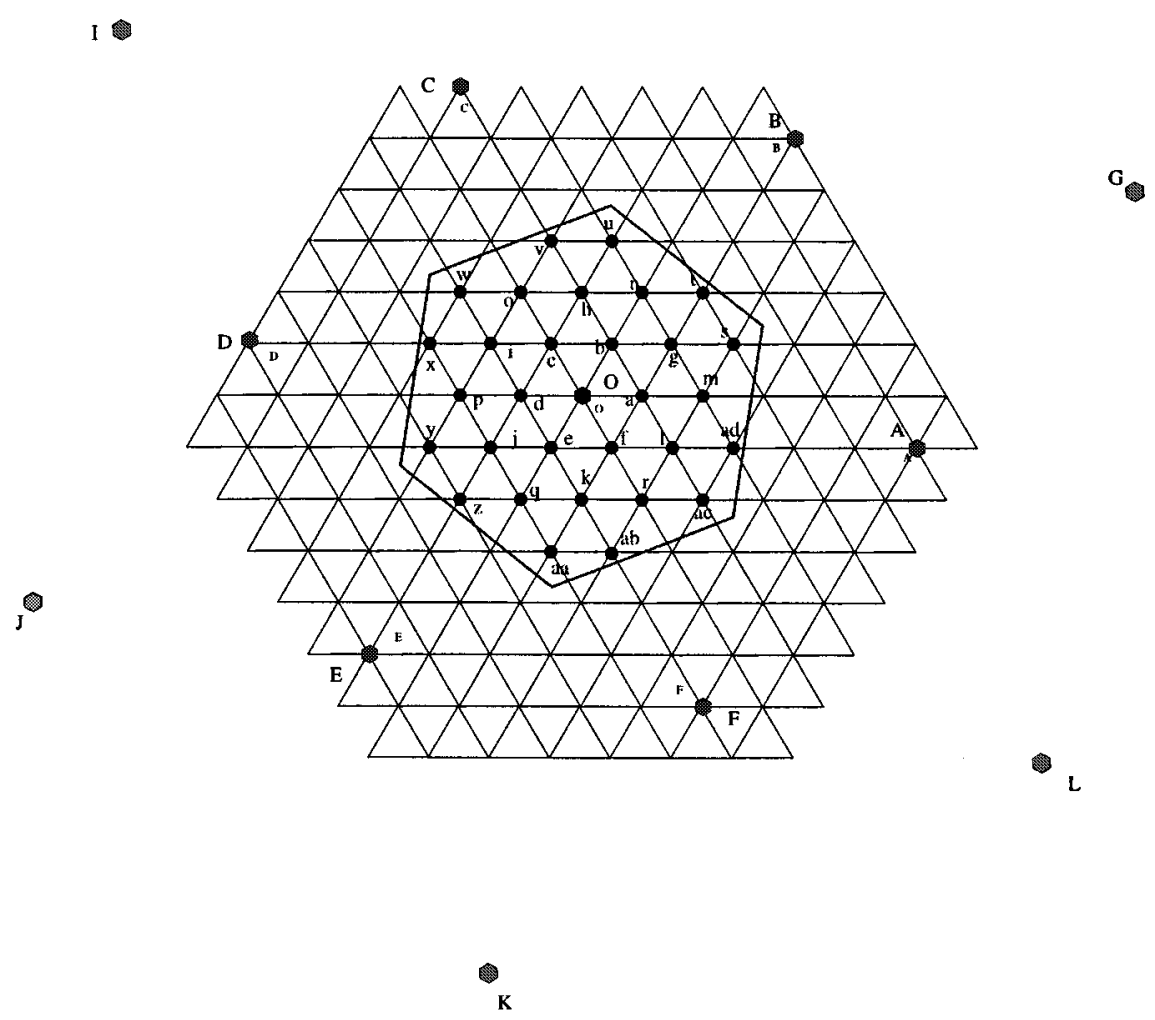

Fig. 5. Example to illustrate the mechanics of the labeling for the hexagonal lattice $A_{2}$.

lattice $A_{2}$-quite explicitly. We will then identify certain general principles and use them to construct labelings for other lattices.

The lattice $A_{2}$ may be considered to be a subset of $\mathbb{R}^{2}$ or as a subset of $\mathbb{C}$. Since each approach has its advantages, we will switch back and forth between the two representations. We consider the lattice $A_{2}$ at unit scale to be generated by the vectors $\{1, \omega\} \subset \mathbb{C}$, where $\omega=-1 / 2+i \sqrt{3} / 2$. The associated Gram matrix is

$$
\left(\begin{array}{cc}
1 & -1 / 2 \\
-1 / 2 & 1
\end{array}\right)
$$

and the fundamental volume is $\sqrt{3} / 2$. A sublattice $\Lambda^{\prime}$ of a lattice $\Lambda$ is said to be geometrically similar to $\Lambda$ if it can be obtained by scaling and rotating and/or reflecting $\Lambda$ [11]. To be more precise, if a matrix $G^{\prime}$ generates $\Lambda^{\prime}$ and $G$ generates $\Lambda$, then $\Lambda^{\prime}$ is geometrically similar to $\Lambda$ if and only if $G^{\prime}=c U G B$, for some nonzero scalar $c$, integer matrix $U$ with determinant \pm 1 , and real orthogonal matrix $B$. The index $N$ is defined as the ratio of the fundamental volumes of $\Lambda^{\prime}$ and $\Lambda$ and is given in terms of the scale factor $c$ by $N=c^{2}$. It can be shown [6] that $\Lambda^{\prime}$ is similar to $\Lambda$ if and only if $N$ is of the form $a^{2}-a b+b^{2}$, $a, b \in \mathbb{Z}$; if this holds, then $\Lambda^{\prime}$ is generated by $\boldsymbol{u}=a+b \omega$ and $\boldsymbol{v}=\omega(a+b \omega)$. In addition to this restriction on $N$, we will require, for convenience only, that $N=\sum_{i=0}^{K} A_{i}$, where $A_{i}$ is the number of lattice points at squared distance $i$ from the origin. In other words, we require that $N$ is the number of points in the first $K$ shells of the lattice, for some $K=K(N)$. There are heuristic arguments, to be presented elsewhere, which suggest that there are infinitely many values of $N$ with this property. For example, $N=31$ has this property, since

$N=A_{0}+A_{1}+A_{2}+A_{3}+A_{4}=1+6+6+6+12=31$ and 31 is also of the form $a^{2}-a b+b^{2}$, with $a=5, b=-1$.

\section{A. An Example}

We now present an example of a labeling function with a reuse index $N=31$. The following are the steps in constructing the labeling function.

1) Find a sublattice with index equal to the reuse index.

2) Determine the discrete Voronoi set $V_{0}(0)$.

3) Determine an undirected label for every point $\lambda \in V_{0}(0)$.

4) Extend the labeling to the entire lattice using the shift property of the undirected labels.

5) Given $\lambda$ and its undirected label $e$, determine the correct directed label $\vec{e}$ (i.e., determine which endpoint of $e$ is to be sent on channel 1 and which endpoint on channel 2).

A sublattice $\Lambda^{\prime}$ of index equal to 31 may be obtained by considering all points of the form $a \boldsymbol{u}+b \boldsymbol{v}$, with $\boldsymbol{u}=5-\omega$ and 


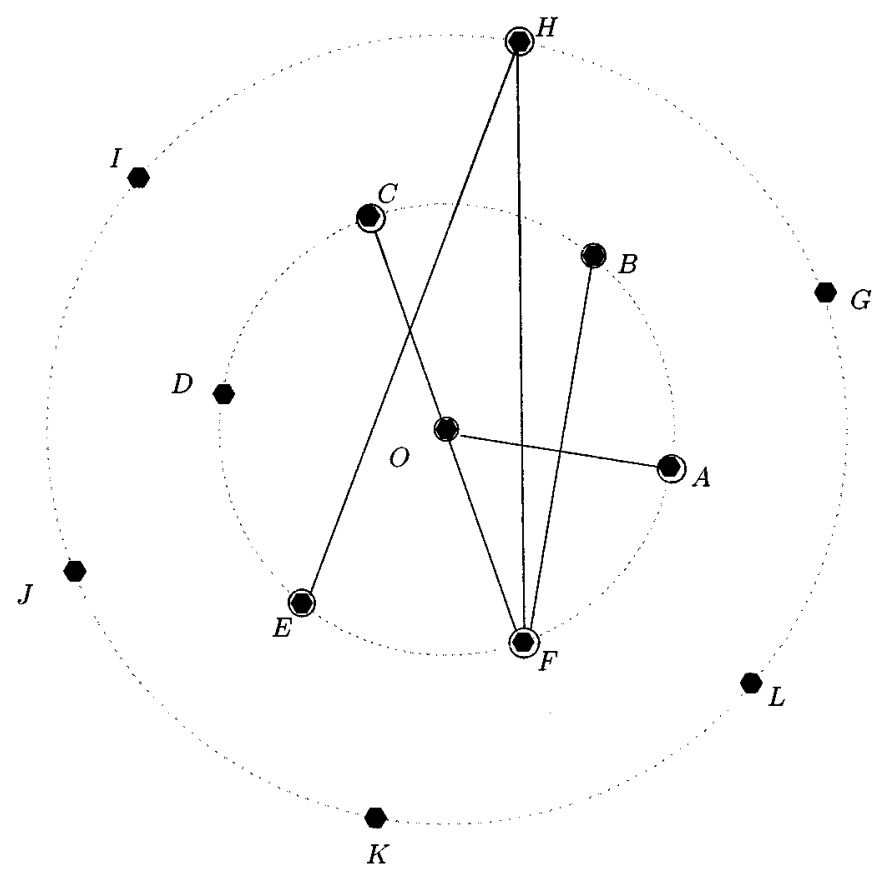

Fig. 6. The set of undirected edges $\mathcal{E}_{u}(0)$ for the example in Section V-A. For clarity, only a subset of $\mathcal{E}_{u}(0)$ is shown. The entire set may be obtained by rotating each edge around the origin (sublattice point $O$ ) by multiples of $\pi / 3$ radians. $\mathcal{E}_{u}(0)$ consists of 28 distinct edges obtained by counting six edges for each edge in the figure, except for the diameter, for which only three edges are counted. The edge $\{O, O\}$ is not shown.

$v=\omega(5-\omega)$. A portion of this lattice together with some sublattice points is shown in Fig. 5. Lattice points have been labeled with lower case letters $a, b, c, \ldots$, and sublattice points with upper case letters $O, A, B, C, \ldots$ In order to fix the coordinate system, note that the sublattice point $O$ is the origin of the complex plane and the lattice points $a$ and $c$ have representations 1 and $\omega$, respectively. Relative to this basis, the representations of sublattice points $A$ and $C$ are $5-\omega$ and $\omega(5-\omega)$, respectively. The discrete Voronoi set

$$
V_{0}(0)=\{O, a, b, c, \ldots, y, z, a a, a b, a c, a d\}
$$

is also shown in Fig. 5. Note that $\left|V_{0}(0)\right|=31$. Points in $V_{0}(0)$ will be labeled using directed edges obtained from the following set of 28 undirected edges:

$$
\begin{aligned}
\mathcal{E}_{u}(0)= & \{O, O\}, \\
& \{O, A\},\{O, B\},\{O, C\},\{O, D\},\{O, E\}, \\
& \{O, F\}, \\
& \{A, C\},\{B, D\},\{C, E\},\{D, F\},\{E, A\}, \\
& \{F, B\}, \\
& \{A, D\},\{B, E\},\{C, F\}, \\
& \{G, D\},\{G, E\},\{H, E\},\{H, F\},\{I, F\}, \\
& \{I, A\}, \\
& \{J, B\},\{J, A\},\{K, C\},\{K, B\},\{L, C\}, \\
& \{L, D\}\}
\end{aligned}
$$

which are illustrated in Fig. 6. Three edges in this set, namely, $\{A, D\},\{B, E\}$, and $\{C, F\}$, will be used twice (i.e., both orientations will be used) in order to obtain 31 labels.

Each point in $V_{0}(0)$ is then associated with an undirected edge in $\mathcal{E}_{u}(0)$ as shown in Table I. This association was done by hand (but see Section V-B2). The only constraint is that equivalent points are mapped to equivalent edges - this can be clearly seen in the table, where equivalent lattice points have been placed in the same row. In order to complete the labeling we need to assign a directed edge to each lattice point based on the color $c(e)(0$ or 1 valued) associated with that edge. If $c(e)=0$, the endpoint of $e$ which is closer to $\lambda$ becomes the channel 1 label and the endpoint which is farther from $\lambda$ becomes the channel 2 label. On the other hand, if $c(e)=1$, the endpoint of $e$ which is closer becomes the channel 2 label and that which is farther becomes the channel 1 label. This is illustrated for two cases-for a point that lies in $V_{0}(0)$ and for a point that lies outside $V_{0}(0)$.

First consider the point $a c=1-2 \omega$ which has been assigned the edge $\{C, L\}=\{1+6 \omega, 4-7 \omega\}$. This edge has midpoint $\mu=5 / 2-1 / 2 \omega$. The color of the edge $\{C, L\}$ is obtained in terms of the first component of the midpoint of the edge $(5 / 2)$ and the difference between the first components of the edge endpoints $(4-1=3)$ by determining whether $\lfloor(5 / 2) / 3\rfloor$ is odd or even. In this case it is even, hence it is assigned the color 0 . (If it had been odd, it would have been assigned the color 1 . An expression for the coloring rule is given later in (22).) The specific orientation of this edge is then obtained by determining which endpoint of $\{C, L\}$ is closer to $a c$. In this case, $L=4-7 \omega$ is closer to $a c$ than $C=1+6 \omega$. Since the edge has color equal to 0 , the closer endpoint becomes the channel 1 label, i.e., $a c$ is assigned the edge $(L, C)$ (this is the edge direction rule (23), which is explained in detail in Section V-B5).

Now consider the lattice point $\lambda=18+10 \omega \notin V_{0}(0)$. The nearest lattice point is $\lambda^{\prime}=17+9 \omega$. We compute the difference $\lambda-\lambda^{\prime}=(1+\omega)$ (the point $b$ ) and use this to look up the corresponding undirected label in Table I. This gives

$$
\alpha_{u}(1+\omega)=\{O, B\}=\{0,6+5 \omega\} .
$$


TABLE I

Labels For Points in the Discrete Voronoi Set $V_{0}(0)$ FOR the HeXagonal Lattice AND Sublattice IndeX $N=31$

\begin{tabular}{|c|c|c|c||c|c|c|c|}
\hline $\begin{array}{c}\text { Lattice } \\
\text { Point } \\
\lambda\end{array}$ & $\begin{array}{c}\text { Label } \\
\text { (undirected) } \\
e=\alpha_{u}(\lambda)\end{array}$ & $\begin{array}{c}\text { Color } \\
c(e)\end{array}$ & $\begin{array}{c}\text { Label } \\
\text { (directed) } \\
\vec{e}=\alpha(\lambda)\end{array}$ & $\begin{array}{c}\text { Lattice } \\
\text { Point } \\
\lambda\end{array}$ & $\begin{array}{c}\text { Label } \\
\text { (undirected) } \\
e=\alpha_{u}(\lambda)\end{array}$ & $\begin{array}{c}\text { Color } \\
c(e)\end{array}$ & $\begin{array}{c}\text { Label } \\
\text { (directed) } \\
\vec{e}=\alpha(\lambda)\end{array}$ \\
\hline \hline $\mathrm{O}$ & $\{\mathrm{O}, \mathrm{O}\}$ & & $(\mathrm{O}, \mathrm{O})$ & & & & \\
$\mathrm{a}$ & $\{\mathrm{O}, \mathrm{A}\}$ & 0 & $(\mathrm{O}, \mathrm{A})$ & $\mathrm{d}$ & $\{\mathrm{O}, \mathrm{D}\}$ & 1 & $(\mathrm{D}, \mathrm{O})$ \\
$\mathrm{b}$ & $\{\mathrm{O}, \mathrm{B}\}$ & 0 & $(\mathrm{O}, \mathrm{B})$ & $\mathrm{e}$ & $\{\mathrm{O}, \mathrm{E}\}$ & 1 & $(\mathrm{E}, \mathrm{O})$ \\
$\mathrm{c}$ & $\{\mathrm{O}, \mathrm{C}\}$ & 0 & $(\mathrm{O}, \mathrm{C})$ & $\mathrm{f}$ & $\{\mathrm{O}, \mathrm{F}\}$ & 1 & $(\mathrm{~F}, \mathrm{O})$ \\
$\mathrm{g}$ & $\{\mathrm{A}, \mathrm{C}\}$ & 0 & $(\mathrm{~A}, \mathrm{C})$ & $\mathrm{j}$ & $\{\mathrm{D}, \mathrm{F}\}$ & 1 & $(\mathrm{~F}, \mathrm{D})$ \\
$\mathrm{h}$ & $\{\mathrm{B}, \mathrm{D}\}$ & 0 & $(\mathrm{~B}, \mathrm{D})$ & $\mathrm{k}$ & $\{\mathrm{E}, \mathrm{A}\}$ & 1 & $(\mathrm{~A}, \mathrm{E})$ \\
$\mathrm{l}$ & $\{\mathrm{F}, \mathrm{B}\}$ & 0 & $(\mathrm{~F}, \mathrm{~B})$ & $\mathrm{i}$ & $\{\mathrm{C}, \mathrm{E}\}$ & 1 & $(\mathrm{E}, \mathrm{C})$ \\
$\mathrm{n}$ & $\{\mathrm{A}, \mathrm{D}\}$ & 0 & $(\mathrm{~A}, \mathrm{D})$ & $\mathrm{q}$ & $\{\mathrm{A}, \mathrm{D}\}$ & 0 & $(\mathrm{D}, \mathrm{A})$ \\
$\mathrm{o}$ & $\{\mathrm{B}, \mathrm{E}\}$ & 0 & $(\mathrm{~B}, \mathrm{E})$ & $\mathrm{r}$ & $\{\mathrm{B}, \mathrm{E}\}$ & 0 & $(\mathrm{E}, \mathrm{B})$ \\
$\mathrm{p}$ & $\{\mathrm{C}, \mathrm{F}\}$ & 0 & $(\mathrm{C}, \mathrm{F})$ & $\mathrm{m}$ & $\{\mathrm{C}, \mathrm{F}\}$ & 0 & $(\mathrm{~F}, \mathrm{C})$ \\
$\mathrm{x}$ & $\{\mathrm{A}, \mathrm{I}\}$ & 0 & $(\mathrm{I}, \mathrm{A})$ & $\mathrm{ad}$ & $\{\mathrm{D}, \mathrm{L}\}$ & 1 & $(\mathrm{D}, \mathrm{L})$ \\
$\mathrm{s}$ & $\{\mathrm{D}, \mathrm{G}\}$ & 0 & $(\mathrm{G}, \mathrm{D})$ & $\mathrm{y}$ & $\{\mathrm{A}, \mathrm{J}\}$ & 1 & $(\mathrm{~A}, \mathrm{~J})$ \\
$\mathrm{t}$ & $\{\mathrm{E}, \mathrm{G}\}$ & 0 & $(\mathrm{G}, \mathrm{E})$ & $\mathrm{z}$ & $\{\mathrm{B}, \mathrm{J}\}$ & 1 & $(\mathrm{~B}, \mathrm{~J})$ \\
$\mathrm{u}$ & $\{\mathrm{E}, \mathrm{H}\}$ & 0 & $(\mathrm{H}, \mathrm{E})$ & $\mathrm{aa}$ & $\{\mathrm{B}, \mathrm{K}\}$ & 1 & $(\mathrm{~B}, \mathrm{~K})$ \\
$\mathrm{v}$ & $\{\mathrm{F}, \mathrm{H}\}$ & 0 & $(\mathrm{H}, \mathrm{F})$ & $\mathrm{ab}$ & $\{\mathrm{C}, \mathrm{K}\}$ & 1 & $(\mathrm{C}, \mathrm{K})$ \\
$\mathrm{ac}$ & $\{\mathrm{C}, \mathrm{L}\}$ & 0 & $(\mathrm{~L}, \mathrm{C})$ & $\mathrm{w}$ & $\{\mathrm{F}, \mathrm{I}\}$ & 1 & $(\mathrm{~F}, \mathrm{I})$ \\
\hline \hline
\end{tabular}

Using the shift property, the undirected label for $\lambda$ is then obtained by shifting the edge by $\lambda^{\prime}$ to give

$$
\alpha_{u}(\lambda)=\{17+9 \omega, 23+14 \omega\} .
$$

To determine the correct edge orientation, we first determine the color of the edge using the first component of the midpoint $((17+23) / 2=20)$ and the first component of the difference $((23-17)=6)$ to obtain a color

$$
c(\{17+9 \omega, 23+14 \omega\})=\lfloor 20 / 6\rfloor(\bmod 2)=1 .
$$

Since the color is 1 , the closer endpoint becomes the channel 2 label. Hence $18+10 \omega$ is assigned the label $(23+14 \omega, 17+9 \omega)$.

We now illustrate the decoding procedure. Assume that $\vec{e}=(23+14 \omega, 17+9 \omega)$ and write its undirected version using the basis vectors of the sublattice $\boldsymbol{u}$ and $\boldsymbol{v}$ to get $\{4 \boldsymbol{u}+3 \boldsymbol{v}, 3 \boldsymbol{u}+2 \boldsymbol{v}\}$. Look for an equivalent edge in Table I. One such edge is $\{O, B\}=\{0, \boldsymbol{u}+\boldsymbol{v}\}$. Determine the shift required to make the edges coincide. In this case $\{4 u+3 v\}-\lambda^{\prime}=\{\boldsymbol{u}+\boldsymbol{v}, 0\}$, with $\lambda^{\prime}=3 u+2 v$. Upon looking up Table I, we find that lattice point $b$ with representation $1+\omega$ has the undirected edge $\{O, B\}$ as label. We shift this point by adding $\lambda^{\prime}=3 u+2 v$ to get one of the candidate points $\lambda=3 \boldsymbol{u}+2 \boldsymbol{v}+(1+\omega)=18+10 \omega$. The other candidate point is obtained from the Property 3 of the labeling function (the sum of the endpoints of an edge is equal to the sum of the points that it labels), and is $22+13 \omega$. Observe that there is another edge in Table I which is equivalent to the edge we wish to decode. We would obtain exactly the same set of candidate points if we used this edge. In order to determine the correct point, since $c(e)=1$, we decode to the point which is closer to the channel 2 label, namely, $18+10 \mathrm{w}$.

Several observations can be made at this point. In Table I there are two kinds of undirected edges (of positive length) - those which are diameters of a circle centered at 0 and those which are not. The diameters are the edges $\{A, D\},\{B, E\}$, and $\{C, F\}$. Both orientations of a diameter are used to label points in $V_{0}(0)$, whereas only one orientation of a nondiameter is used. For an edge which is not a diameter, the remaining orientation labels a lattice point outside $V_{0}(0)$ as determined by the shift property. For example, consider the label $\{L, C\}$. The directed label $(L, C)$ is the label for the point $a c=1-2 \omega \in V_{0}(0)$. The lattice point which is labeled by $(C, L)$ is given by

$$
2 \mu-(1-2 \omega)=5-\omega-(1-2 \omega)=4+\omega
$$

which belongs to the discrete Voronoi set of the sublattice point A.

Notice that the labeling function shown in Table I exhibits an additional symmetry that we have so far not used. If a point is rotated by a multiple of $\pi / 3$ radians about the origin, its corresponding undirected label is also rotated by the same amount about the origin. Consider

$$
\Gamma=\left\{\gamma_{i}=\exp i k \pi / 3, k=0,1, \ldots, 5\right\}
$$

a rotation group of order 6 . By considering equivalence classes relative to this group, we can reduce the size of the table by listing only the undirected edges for the points $\{o, a, g, n, t, s\}$.

The above example illustrates the basic steps that are to be followed in order to label the points in $\Lambda$. Additional details and some underlying theory are presented next.

\section{B. General Principles}

The construction of our labeling function involves the following steps.

S1) Selection of a geometrically similar sublattice of given index $N$.

S2) Construction of $V_{0}(0)$, the discrete Voronoi set around 0 .

S3) Establishing a mapping between elements of $V_{0}(0)$ and undirected edges in such a way that certain constraints are satisfied. The optimal construction requires that a specific linear programming problem be solved.

S4) Extension of the mapping to the entire lattice.

S5) Identification of a specific directed edge to associate with a lattice point, once the undirected edge is known. 


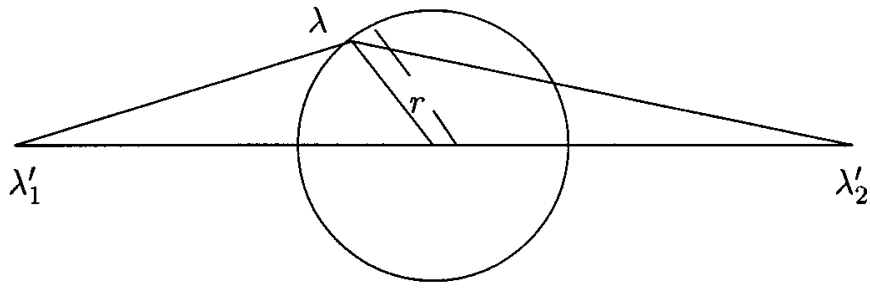

Fig. 7. A lattice point $\lambda$ and its label $\left(\lambda_{1}^{\prime}, \lambda_{2}^{\prime}\right) . \lambda$ is at distance $r$ from the midpoint of the labels.

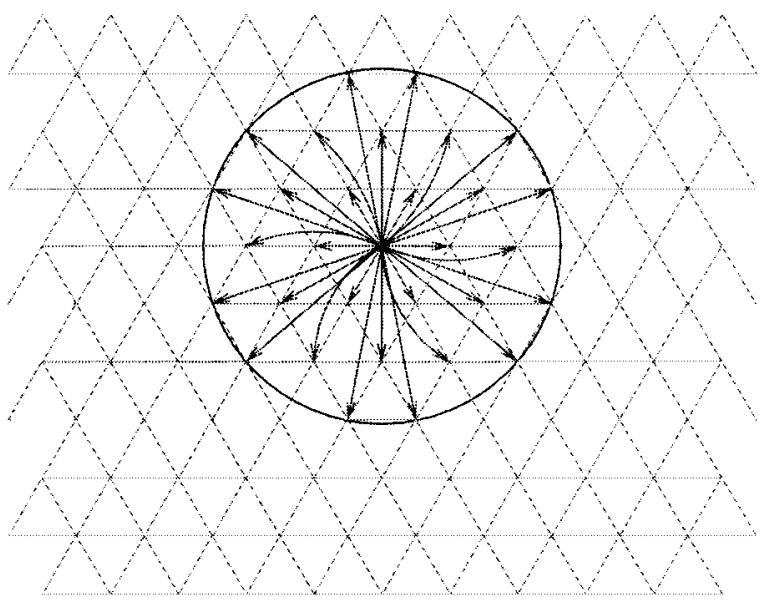

Fig. 8. The set $\left.\mathcal{E}_{d}\right|_{\alpha_{1}=0}$ for $N=31$.

In the remainder of this section, we will further describe steps S3)-S5). But first we state the following guiding principle.

1) A Guiding Principle: Suppose that $\lambda$ receives the label $\vec{e}=\left(\lambda_{1}^{\prime}, \lambda_{2}^{\prime}\right)$ as illustrated in Fig. 7. Then $d_{s}(\vec{e})$ satisfies the identity

$$
\begin{aligned}
2 d_{s}(\vec{e}) & =\left\|\lambda-\lambda_{1}^{\prime}\right\|^{2}+\left\|\lambda-\lambda_{2}^{\prime}\right\|^{2} \\
& =(1 / 2)\left\|\lambda_{1}^{\prime}-\lambda_{2}^{\prime}\right\|^{2}+2 r^{2}
\end{aligned}
$$

where $r^{2}=\left\|\lambda-\left(\lambda_{1}^{\prime}+\lambda_{2}^{\prime}\right) / 2\right\|^{2}$. This identity is known as the parallelogram law (see, for example, [2, p. 3]). From this we infer that in order to keep $\bar{d}_{s}(\vec{e})$ small, $\lambda_{1}^{\prime}$ and $\lambda_{2}^{\prime}$ should be as close together as possible, and $\lambda$ should be as close as possible to their midpoint. This leads to the following:

Guiding Principle: Choose the shortest possible edge with midpoint as close as possible to the point to be labeled.

2) Optimal Construction of $\mathcal{E}_{u}(0)$ and $\alpha_{u}$ (steps S3) and S4)): The starting point for determining the set of edges is to compute $\left.\mathcal{E}_{d}\right|_{\alpha_{1}=0}$, which is chosen to be the set of $N$ shortest undirected sublattice edges of the form $\left(0, \lambda^{\prime}\right)$. This set is illustrated in Fig. 8. The corresponding set $\left.\mathcal{E}_{u}\right|_{\alpha_{1}=0}$ is obtained by replacing each directed edge with its undirected version. Observe that edges of positive length in $\left.\mathcal{E}_{u}\right|_{\alpha_{1}=0}$ occur in equivalent pairs. Next, for every $\lambda \in V_{0}(0)$ and $\left.e \in \mathcal{E}_{u}\right|_{\alpha_{1}=0}$, determine

$$
d_{s}(\lambda,[e])=\min _{\tilde{e} \in[e]} d_{s}(\lambda, \tilde{e})
$$

and let $\alpha^{*}(\lambda,[e])$ be an edge $\tilde{e} \in[e]$ which achieves this minimum. In other words, $\alpha^{*}(\lambda,[e])$ is the "closest" edge to $\lambda$ which lies in the same equivalence class as $e$. Clearly, from the guiding principle, the "closest" edge will be the one whose midpoint is closest to $\lambda$. The edge $\alpha^{*}(\lambda,[e])$ for a given $\lambda$ and $e$ is illustrated in Fig. 9.

Consider all one-to-one maps $\beta:\left.V_{0}(0) \rightarrow \mathcal{E}_{u}\right|_{\alpha_{1}=0}$ which satisfy the constraint that equivalent points are mapped to equivalent edges. From among all such maps $\beta$, choose $\beta^{*}$ so as to minimize $\sum_{\lambda \in V_{0}(0)} d_{s}(\lambda,[\beta(\lambda)])$. The map $\beta^{*}$ sends a lattice point to an edge coset element in $\left.\mathcal{E}_{u}\right|_{\alpha_{1}=0}$ in an optimal way, thus identifying the best edge coset for a given lattice point. Let $\alpha_{u}(\lambda)=\alpha^{*}\left(\lambda,\left[\beta^{*}(\lambda)\right]\right)$. Since $\beta^{*}$ identifies the optimal edge coset for each lattice point and $\alpha^{*}$ identifies the best coset representative, given the lattice point and the coset, we obtain the optimal edge for each lattice point by composing these two mappings. It follows that $\mathcal{E}_{u}(0)=\left\{\alpha_{u}(\lambda): \lambda \in V_{0}(0)\right\}$. We extend the mapping to the lattice $\Lambda$ using the rule $\alpha_{u}\left(\lambda+\lambda^{\prime}\right)=$ $\alpha_{u}(\lambda)+\lambda^{\prime}$.

The constraint imposed on the mapping $\beta$ needs some explanation. It arises from the third assumption that we made about the labeling function, namely, that the sum of the endpoints of an edge is equal to the sum of the two points that it labels. By requiring that equivalent points map to equivalent edges, it can be shown that the midpoint of two lattice points that share the same (undirected) label coincides with the midpoint of the label itself. A graphical justification for this is provided in Fig. 10. More formally, the argument is as follows. The points in $V_{0}(0)$ occur in equivalent pairs. If two points in $V_{0}(0)$ are equivalent, they sum to 0 . Consider the pair $\lambda_{a}$ and $-\lambda_{a}$ and an edge $e$. If the edge in $[e]$ which is closest to $\lambda_{a}$, say $e_{a}$, has midpoint $a$, then the edge in $[e]$ which is closest to $-\lambda_{a}$ will have midpoint $-a$. Thus, $\mathcal{E}_{u}(0)$ contains the edges $e_{a}$ and $-e_{a}$ (which may be identical). Now from the shift property, $e_{a}$ also lies in $\mathcal{E}_{u}(2 a)=\mathcal{E}_{u}(0)+2 a$ (note that $2 a$ is a sublattice point) and the point it labels, say $\lambda_{b}$, is given by $\lambda_{b}=-\lambda_{a}+2 a$. Thus, the two points that are labeled using the undirected edge $e_{a}$, namely, $\lambda_{a}$ and $\lambda_{b}$, satisfy $\lambda_{a}+\lambda_{b}=2 a$, i.e., the midpoint of the edge coincides with the midpoint of the points that receive this edge as label. To summarize, the constraint is a sufficient condition to ensure that the labeling function has Property 3 ).

It is to be noted that the optimal mapping $\beta^{*}$ can be obtained using standard techniques from linear programming [21]. Also observe that if we define the group $\Gamma=\{1,-1\}$, we can force $\beta^{*}$ to satisfy the constraint by considering only $V_{0}(0) / \Gamma$ and $\left.\mathcal{E}_{u}\right|_{\alpha_{1}=0} / \Gamma$. This problem is one of matching $(N-1) / 2$ lattice points to $(N-1) / 2$ edge classes. Further reductions in complexity may be obtained by using a larger group $\Gamma$, in which case the problem is reduced to matching $(N-1) / o(\Gamma)$ points to $(N-1) / o(\Gamma)$ edge classes, where $o(\Gamma)$ is the order of the group. In the case of the $A_{2}$ lattice, for example, we could take $\Gamma$ to be a group of order 6 .

The mapping $\alpha_{u}$ identifies the edge to be associated with $\lambda$ up to the orientation of the edge. The correct orientation is determined by an edge orientation rule, designed to maintain balance between the two descriptions. This is described next.

3) Balance: Given an edge $e$ and two points $\left\{\lambda_{a}, \lambda_{b}\right\}$ that it labels, there are two ways to establish a one-to-one correspondence between the points and the two directed edges $\{\vec{e}, \overleftarrow{e}\}$, as described in Fig. 11. The first selection rule favors the second 


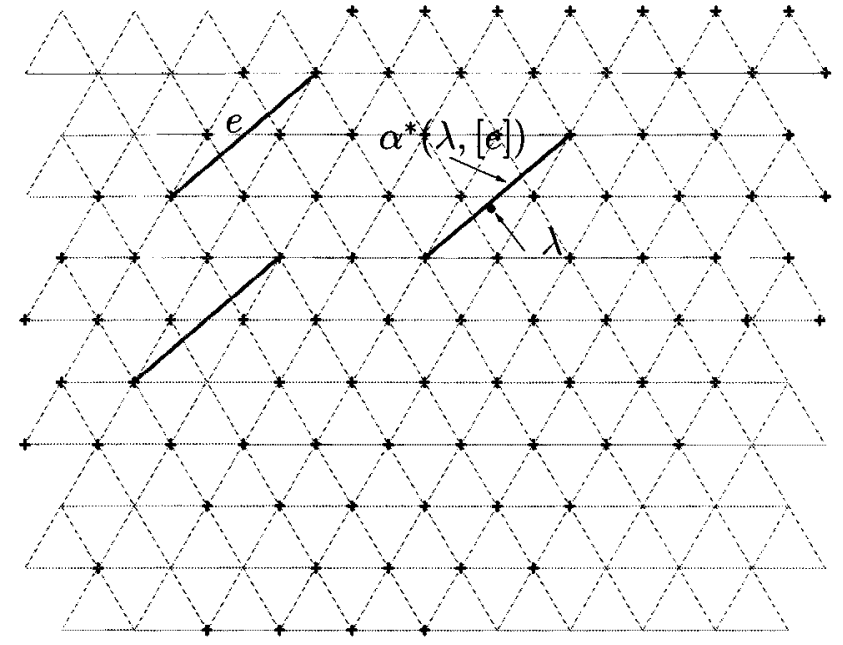

Fig. 9. Illustrating the optimal edge selection within a given equivalence class. Shown is an edge $e$, another edge in its equivalence class $[e]$, and the optimal edge in this class for labeling the point $\lambda$. The points on the hexagonal grid are sublattice points.

description, the second favors the first description. Note that the distortions are antisymmetric, i.e., $d_{1}(e)$ with the first selection rule is equal to $d_{2}(e)$ for the second, and $d_{2}(e)$ for the first rule is equal to $d_{1}(e)$ for the second. Because of this antisymmetry, balance is attainable in an average sense by ensuring that the two correspondences are used equally often. We achieve this by requiring that the two correspondences are alternated along any straight line of the edge graph, as shown in Fig. 12. This is the purpose of the edge-coloring rule which is described next.

4) An Edge Coloring Rule: Each edge $e$ is assigned a bit (or color $\left.^{2}\right) c(e)$ as follows. Consider an edge of the form $(a+b \omega$, $c+d \omega)$. Let $\Delta_{1}=|c-a|$ and let $\Delta_{2}=|d-b|$. Then

$$
c(e)= \begin{cases}0, & \text { if } \Delta_{1}>0 \text { and }\left\lfloor(c+a) /\left(2 \Delta_{1}\right)\right\rfloor \text { is even } \\ 1, & \text { or if } \Delta_{1}=0 \text { and }\left\lfloor(d+b) /\left(2 \Delta_{2}\right)\right\rfloor \text { is even } \\ \text { otherwise. }\end{cases}
$$

This coloring rule ensures that adjacent edges along any straight line have a different color. Note that the color does not depend on the orientation of an edge, i.e., $\vec{e}, \overleftarrow{e}$, and $e$ have the same color. The coloring rule is an ingredient in the edge and point selection rules that we now define.

5) Edge Direction Rule and Point Selection Rule: Given an (undirected) edge $e$ and a point $\lambda$ for which this edge is a label, we choose an orientation or direction for the edge using a rule that depends on the color of the edge. Let $e=\left\{\lambda_{1}^{\prime}, \lambda_{2}^{\prime}\right\}$ and let $\mu=\left(\lambda_{1}^{\prime}+\lambda_{2}^{\prime}\right) / 2$. The two rules $s_{c}(e, \lambda)$, where $c=0$ or 1 is the color of the edge, are defined as follows $(x$ denotes the crossor vector-product of two vectors and $\langle\cdot, \cdot\rangle$ their inner product):

$$
s_{0}(e, \lambda)= \begin{cases}\left(\lambda_{1}^{\prime}, \lambda_{2}^{\prime}\right), & \left\langle\lambda_{1}^{\prime}-\lambda_{2}^{\prime}, \lambda-\mu\right\rangle>0 \text { or } \\ & \left\langle\lambda_{1}^{\prime}-\lambda_{2}^{\prime}, \lambda-\mu\right\rangle=0 \text { and } \\ & \text { sign }\left(\left(\lambda_{1}^{\prime}-\lambda_{2}^{\prime}\right) \times(\lambda-\mu)\right)>0 \\ \left(\lambda_{2}^{\prime}, \lambda_{1}^{\prime}\right), & \text { otherwise }\end{cases}
$$

and

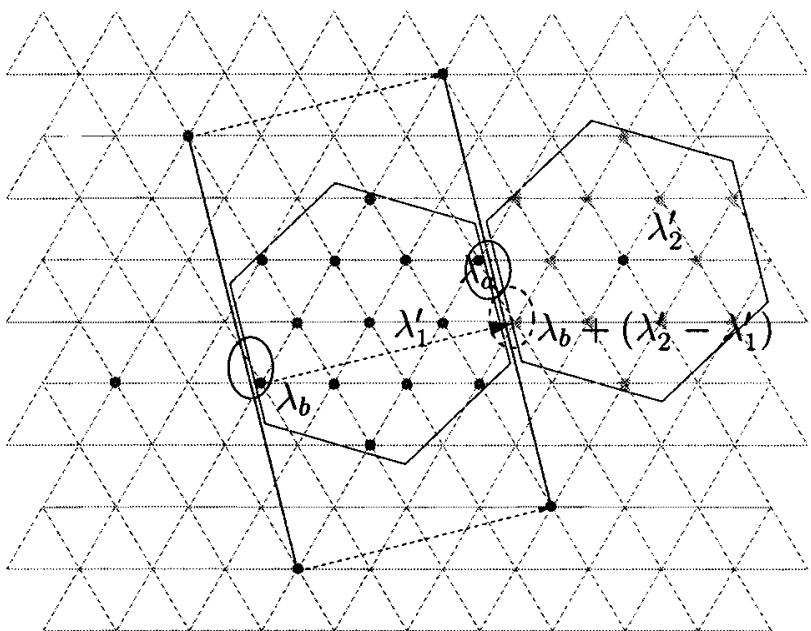

Fig. 10. Explanation of reason for mapping equivalent points in $V_{0}(0)$ to equivalent edges. The lattice $A_{2}$ together with a similar sublattice of index $N=13$ is shown. Points $\lambda_{a}$ and $\lambda_{b}$ in $V_{0}\left(\lambda_{1}^{\prime}\right)$ are equivalent and $\lambda_{a}+\lambda_{b}=2 \lambda_{1}^{\prime} \cdot \lambda_{a}$ and $\lambda_{b}$ are mapped to equivalent edges $e_{a}$ and $e_{b}$. The edges $e_{a}$ and $e_{b}$ have midpoints $\mu_{a}$ and $\mu_{b}$, respectively, which satisfy $\mu_{a}+\mu_{b}=2 \lambda_{1}^{\prime}$ and $e_{a}=e_{b}+\left(\lambda_{2}^{\prime}-\lambda_{1}^{\prime}\right)$. The point $\lambda_{b}+\left(\lambda_{2}^{\prime}-\lambda_{1}^{\prime}\right)$ lies in $V_{0}\left(\lambda_{2}^{\prime}\right)$ and is labeled by $e_{b}+\left(\lambda_{2}^{\prime}-\lambda_{1}^{\prime}\right)=e_{a}$. Thus, $\lambda_{a}+\left(\lambda_{b}+\left(\lambda_{2}^{\prime}-\lambda_{1}^{\prime}\right)\right)=\lambda_{1}^{\prime}+\lambda_{2}^{\prime}=\mu_{a}+\mu_{b}$. The midpoint of two points labeled by the same undirected edge is equal to the midpoint of the edge.

$s_{1}(e, \lambda)= \begin{cases}\left(\lambda_{2}^{\prime}, \lambda_{1}^{\prime}\right), & \left\langle\lambda_{1}^{\prime}-\lambda_{2}^{\prime}, \lambda-\mu\right\rangle>0 \text { or } \\ & \left\langle\lambda_{1}^{\prime}-\lambda_{2}^{\prime}, \lambda-\mu\right\rangle=0 \text { and } \\ & \text { sign }\left(\left(\lambda_{1}^{\prime}-\lambda_{2}^{\prime}\right) \times(\lambda-\mu)\right)>0 \\ \left(\lambda_{1}^{\prime}, \lambda_{2}^{\prime}\right), & \text { otherwise. }\end{cases}$

Observe that the result of either rule is the same whether we write $e=\left\{\lambda_{1}^{\prime}, \lambda_{2}^{\prime}\right\}$ or $e=\left\{\lambda_{2}^{\prime}, \lambda_{1}^{\prime}\right\}$.

For decoding, since two lattice points receive a label from a given undirected edge, we need to be able to tell which point is being labeled, given the edge orientation. This is the reverse of the edge direction rule. Thus, given a directed edge $\vec{e}=$ $\left(\lambda_{1}^{\prime}, \lambda_{2}^{\prime}\right)$ with midpoint $\mu$ and a lattice point $\lambda$ which could have received this label, the Point Selection Rule $g_{c}(\vec{e}, \lambda)$ selects $\lambda$ or $2 \mu-\lambda$ based on the edge color $c$, and is given by

$g_{0}(\vec{e}, \lambda)= \begin{cases}\lambda, \quad & \left\langle\lambda_{1}^{\prime}-\lambda_{2}^{\prime}, \lambda-\mu\right\rangle>0 \text { or } \\ & \left\langle\lambda_{1}^{\prime}-\lambda_{2}^{\prime}, \lambda-\mu\right\rangle=0 \text { and } \\ & \text { sign }\left(\left(\lambda_{1}^{\prime}-\lambda_{2}^{\prime}\right) \times(\lambda-\mu)\right)>0 \\ 2 \mu-\lambda & \text { otherwise }\end{cases}$

and

$g_{1}(\vec{e}, \lambda)= \begin{cases}2 \mu-\lambda, & \left\langle\lambda_{1}^{\prime}-\lambda_{2}^{\prime}, \lambda-\mu\right\rangle>0 \text { or } \\ & \left\langle\lambda_{1}^{\prime}-\lambda_{2}^{\prime}, \lambda-\mu\right\rangle=0 \text { and } \\ & \text { sign }\left(\left(\lambda_{1}^{\prime}-\lambda_{2}^{\prime}\right) \times(\lambda-\mu)\right)>0 \\ & \text { otherwise. }\end{cases}$

6) Constructing the Map $\alpha$ : Using the notation previously established, we obtain $\alpha$ as follows. Given $\lambda$, let $e=\alpha_{u}(\lambda)$ and let $c=c(e)$. Then $\alpha(\lambda)=s_{c}(e, \lambda)$.

7) Proof that the Reuse Index is Correct: We need to show that for any sublattice point $\lambda^{\prime}$, exactly $N$ lattice points have a label of the form $\left(\lambda^{\prime}, *\right)$ and exactly $N$ lattice points have a label of the form $\left(*, \lambda^{\prime}\right)$.

To begin, observe i) that if edge $e$ lies in $\mathcal{E}_{u \mid \alpha_{1}=0}$ then so does $-e$ (note that $N$ is odd) and ii) $\left|\mathcal{E}_{u \mid \alpha_{1}=0}\right|=N$. Define

$$
I\left(\lambda^{\prime}, \lambda^{\prime \prime}\right)= \begin{cases}1, & \left\{\lambda^{\prime}, \lambda^{\prime \prime}\right\} \in \mathcal{E}_{u} \\ 0, & \text { otherwise. }\end{cases}
$$



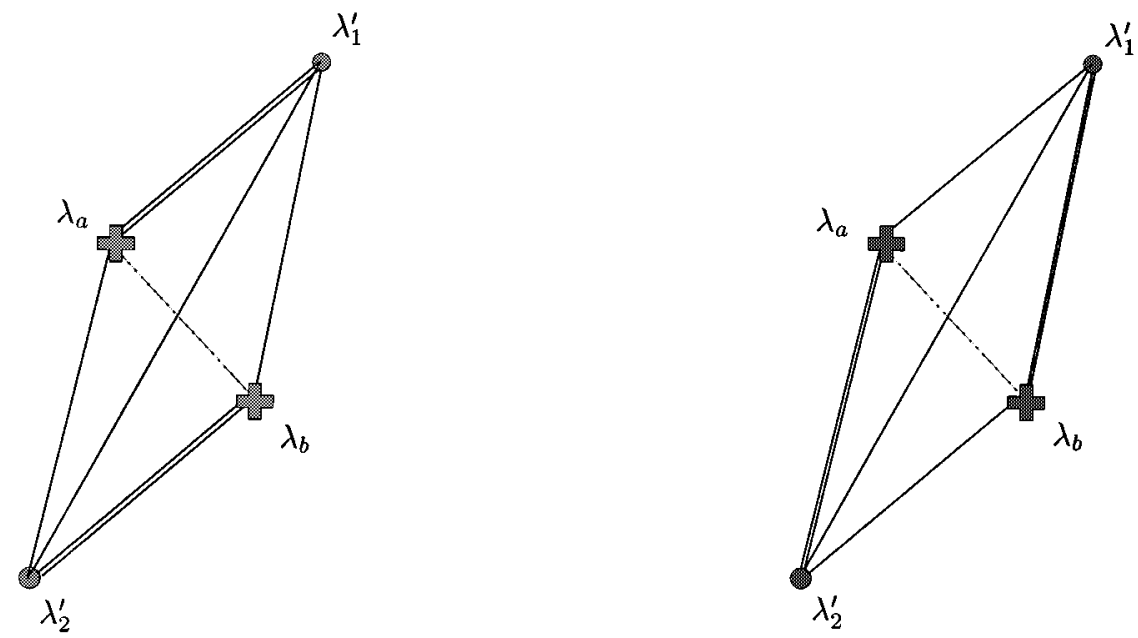

Fig. 11. Given an edge $e=\left\{\lambda_{1}^{\prime}, \lambda_{2}^{\prime}\right\}$ and the two lattice points $\left\{\lambda_{a}, \lambda_{b}\right\}$ that it labels, with the midpoint of $e$ coinciding with the midpoint $\left(\lambda_{a}+\lambda_{b}\right) / 2$, there are two possibilities for the selection rule. In the figure, the lattice points are joined to the sublattice points by both a single line, indicating the first component, and a double line, indicating the second component. On the left, the double lines are shorter than the single lines, a selection rule which favors the second description, that is, $d_{2}$ is smaller than $d_{1}$. On the right, the single lines are shorter than the double lines, a rule which favors the first description. Balance is achieved by requiring that both rules are used equally often.

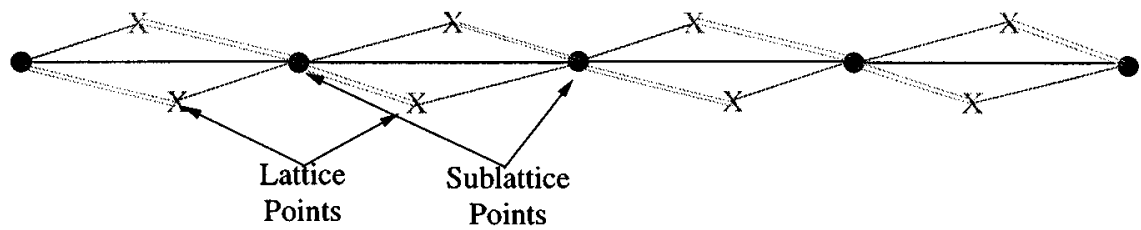

Fig. 12. An illustration of the alternating structure along a straight line for maintaining balance between the two descriptions. A single line connecting a lattice point to a sublattice point indicates the channel 1 label for that lattice point, a double line indicates the channel 2 label.

Then $\sum_{\lambda^{\prime} \in \Lambda^{\prime}} I\left(0, \lambda^{\prime}\right) \geq N$, since $\left\{0, \lambda^{\prime}\right\} \in \mathcal{E}_{u \mid \alpha_{1}=0}$ implies $I\left(0, \lambda^{\prime}\right)=1$. Conversely, if $I\left(0, \lambda^{\prime}\right)=1$, then $\left\{\lambda,{ }^{\prime \prime} \lambda^{\prime}+\lambda^{\prime \prime}\right\} \in$ $\mathcal{E}_{u \mid \alpha_{1}=0}$, for some $\lambda^{\prime \prime}$. Thus, either $\lambda^{\prime \prime}=0$, which implies $\left\{0, \lambda^{\prime}\right\} \in \mathcal{E}_{u \mid \alpha_{1}=0}$ or $\lambda^{\prime \prime}=-\lambda^{\prime}$, which implies $\left\{0,-\lambda^{\prime}\right\} \in$ $\mathcal{E}_{u \mid \alpha_{1}=0}$ which in turn implies that $\left\{0, \lambda^{\prime}\right\} \in \mathcal{E}_{u \mid \alpha_{1}=0}$. Thus

$$
\sum_{\lambda^{\prime} \in \Lambda^{\prime}} I\left(0, \lambda^{\prime}\right)=N
$$

Furthermore, for any $\lambda^{\prime} \in \Lambda^{\prime}$

$$
\sum_{\lambda^{\prime \prime} \in \Lambda^{\prime}} I\left(\lambda^{\prime}, \lambda^{\prime \prime}\right)=\sum_{\lambda^{\prime \prime} \in \Lambda^{\prime}} I\left(0, \lambda^{\prime \prime}-\lambda^{\prime}\right)=N
$$

since the labeling is extended through sublattice shifts. Thus, for any $\lambda^{\prime} \in \Lambda^{\prime}$, there are exactly $N$ edges of the form $\left\{\lambda^{\prime}, *\right\}$.

Each edge $\left\{\lambda^{\prime}, \lambda^{\prime \prime}\right\}$ of positive length labels two points, of which one receives the label $\left(\lambda^{\prime}, \lambda^{\prime \prime}\right)$ the other $\left(\lambda^{\prime \prime}, \lambda^{\prime}\right)$. Thus, exactly $N$ lattice points receive a label of the form $\left(\lambda^{\prime}, *\right)$ and exactly $N$ receive a label of the form $\left(*, \lambda^{\prime}\right)$.

8) Further Reduction in Complexity: Group Construction Instead of imposing the constraint on the map $\beta$ that was used in Section V-B2, we could alternatively regard this map as an unconstrained map between cosets $\Lambda / \Lambda^{\prime} / \Gamma$ and $\mathcal{E}_{u} / \Lambda^{\prime} / \Gamma$, where $\Gamma$ is the group of rotations $\{1,-1\}$ (in complex notation since we are talking about $A_{2}$ ). Now we need only establish a correspondence between two sets of size $(N-1) / 2$, where
2 is the order of the group. Further reductions in complexity arise from selecting a larger group that contains the group $\{1,-1\}$ as a subgroup. For $A_{2}$, we used the group of rotations $\{\exp (i k \pi / 6), k=0,1,2,3,4,5\}$. This reduces the complexity of matching problem to sets of size $(N-1) / 6$. Precise conditions that the group must satisfy, and further reasons for using a group, are explained in Section VI.

9) Numerical Results: The results of our optimization procedure for the hexagonal lattice $A_{2}$ are displayed in Fig. 13, along with comparisons with the $\mathbb{Z}$ lattice. These results have been obtained for a uniformly distributed memoryless source by computing the optimal labeling function and then evaluating the expression (obtained from (15) for a uniform pdf)

$$
\bar{d}_{s}=\bar{d}_{0}+(1 / N) \sum_{\lambda \in V_{0}(0)} d_{s}(e)
$$

where $e$ is the edge that labels $\lambda, \bar{d}_{0}$ is computed using (10) and known values of $G(\Lambda)$ [11]. To be comparable with $A_{2}$, the value of $N$ shown for the $\mathbb{Z}$ lattice is the square of the actual reuse index for dimension $L=1$. To be more specific, let $N_{A_{2}}$ denote the index for the $A_{2}$ sublattice, and let $N_{\mathbb{Z}}$ be the index of the sublattice of $\mathbb{Z}$. Then the value of $N$ stated in the figure is $N_{\mathbb{Z}}^{2}$ for $\mathbb{Z}$ and $N_{A_{2}}$ for $A_{2}$. Also it should be noted that for each $N$, both lattices have been scaled in order to keep the product $N \nu$ constant, where $\nu$ is the volume of a fundamental region of the scaled lattice. From (17), this is equivalent to keeping the rate constant for all points on the graph. It is seen that small 


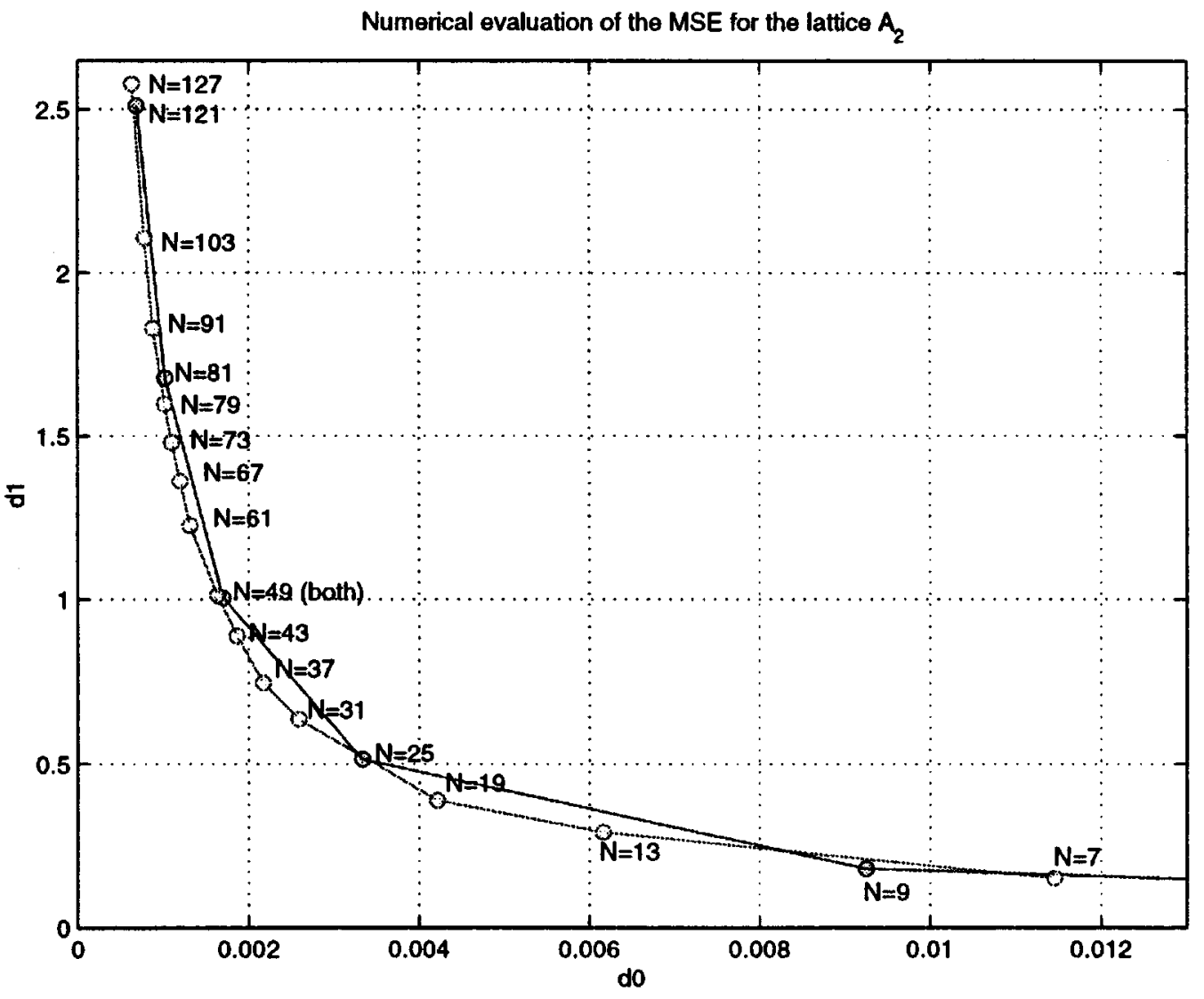

Fig. 13. A plot of $\bar{d}_{1}$ versus $\bar{d}_{0}$ for the hexagonal lattice $A_{2}$ and the integer lattice $\mathbb{Z}$.

performance improvements are obtained by using $A_{2}$ instead of $\mathbb{Z}$. Another benefit is that we obtain many more points in a given interval of (say) the side distortion, compared with the $\mathbb{Z}$ lattice.

\section{LABELING FUnCTIONS FOR GENERAL LATTICES}

In this section, we describe how to label a general lattice $\Lambda$ using a sublattice $\Lambda^{\prime}$ of index $\left[\Lambda: \Lambda^{\prime}\right]=N$. The basic steps remain the same as for $A_{2}$. The main differences arise in the selection of the sublattice and in the use of a group to simplify the construction. We begin by establishing certain general conditions that the group should satisfy. Specific groups and sublattices will then be given for certain particular lattices. We will use $\boldsymbol{G}$ to denote a generator matrix for $\Lambda$ and $\tilde{C} \tilde{G} \boldsymbol{G}$ for the generator matrix for the similar sublattice $\Lambda^{\prime}$, where $c$ is a scalar and $\tilde{\boldsymbol{G}}$ is a unitary matrix. Our convention is that the columns of a generator matrix are a basis for the lattice.

\section{A. Sufficient Conditions for the Group $\Gamma$}

The smallest group we can use is $\Gamma=\left\{I_{L},-I_{L}\right\}$, where $I_{L}$ is the $L$-dimensional identity matrix. Here we show why a group is useful for reducing the size of the optimization problem and derive certain conditions that the group should satisfy.

The motivation for using a group is to make use of inherent symmetries in the lattice and sublattice. Our objective is to partition the discrete Voronoi set and the edge set into subsets of equal size with certain distance properties. More specifically, for any subset of lattice points $\left\{\lambda_{i}, i=1,2, \ldots, M\right\}$ in this partition and any subset of edges $\left\{e_{i}, i=1,2, \ldots, M\right\}$ in the partition the set of distances

$$
\mathcal{D}_{i}=\left\{d_{s}\left(\lambda_{i}, e_{j}\right), j=1,2, \ldots, M\right\}
$$

should be independent of $i$. Such sets of points and edges can be obtained by identifying a group of transformations $\Gamma=\left\{\gamma_{k}, k=1,2, \ldots, M\right\}$ and then taking the members of the partition to be orbits under the action of this group. The group we look for should have following properties.

1) $\Gamma$ contains $-I_{L}$.

2) $\Gamma$ is an orthogonal group.

3) $\Gamma$ preserves the lattice $\Lambda$.

4) $\Gamma$ acts fixed-point free on $\mathbb{R}^{L}$, i.e., for any $\gamma \in \Gamma, \gamma$ not the identity, $\gamma x=x \Longrightarrow x=0$.

5) The order of the group divides the g.c.d. of all the shell sizes.

6) $\Gamma$ preserves the sublattice $\Lambda^{\prime}$.

Property 2) ensures that orbits of the group lie entirely within a shell of the lattice. Property 3) is obvious, otherwise orbits would contain points other than lattice points. Property 4) ensures that all orbits are of equal size. Property 5) ensures that each shell is partitioned into an integral number of orbits. Property 6) is similar to Property 3). 
We now look at Properties 3) and 6) more closely. Every lattice point $\lambda$ can be written in terms of the generator matrix $\boldsymbol{G}$ and an integer vector $u$ as $\lambda=\boldsymbol{G} u$. By requiring that $\gamma_{i} \boldsymbol{G} u=\boldsymbol{G} \gamma_{j} u$ for some $\gamma_{j} \in \Gamma$, we ensure that the lattice is preserved. Similarly, if the sublattice has generator matrix $\tilde{C} \tilde{G} \boldsymbol{G}$, where $c$ is a scalar, then by requiring $\gamma_{i} \tilde{\boldsymbol{G}}=\tilde{\boldsymbol{G}} \gamma_{j}$, for some $\gamma_{j} \in \Gamma$, the sublattice will also be preserved. In other words, we require that $\Gamma$ normalizes $\boldsymbol{G}$ and $\tilde{\boldsymbol{G}}$.

\section{B. Group Construction and Generator Matrices}

For the space lattice $\mathbb{Z}^{2}$, we take $N$ to be an odd number of the form $a^{2}+b^{2}$ where $a, b \in \mathbb{Z}$. (It is shown in [8] that similar sublattices of $\mathbb{Z}^{2}$ exist whenever $N$ is a sum of two squares.) The generator matrices are $\boldsymbol{G}=I_{2}$ and $\boldsymbol{G}^{\prime}=\tilde{\boldsymbol{G}} \boldsymbol{G}$, where

$$
\tilde{\boldsymbol{G}}=\left(\begin{array}{rr}
a & -b \\
b & a
\end{array}\right)
$$

For the group we use

$$
\Gamma=\left\{ \pm I_{2}, \pm\left(\begin{array}{rr}
0 & -1 \\
1 & 0
\end{array}\right)\right\}
$$

a group of order 4 .

For the lattice $\mathbb{Z}^{4}$ we take $N$ to be an odd perfect square (again see [8]). Let $N=a^{2}+b^{2}+c^{2}+d^{2}, a, b, c, d \in \mathbb{Z}$ (any integer can be written this way). The generator matrices are $I_{4}$ and $\boldsymbol{G}^{\prime}=\tilde{G} \boldsymbol{G}$, with

$$
\tilde{G}=\left(\begin{array}{rrrr}
a & -b & -c & -d \\
b & a & -d & c \\
c & d & a & -b \\
d & -c & b & a
\end{array}\right)
$$

The group $\Gamma$ is

$$
\begin{aligned}
\Gamma= & \pm I_{4}, \pm\left(\begin{array}{rrrr}
0 & -1 & 0 & 0 \\
1 & 0 & 0 & 0 \\
0 & 0 & 0 & 1 \\
0 & 0 & -1 & 0
\end{array}\right), \\
& \pm\left(\begin{array}{rrrr}
0 & 0 & -1 & 0 \\
0 & 0 & 0 & -1 \\
1 & 0 & 0 & 0 \\
0 & 1 & 0 & 0
\end{array}\right) \\
& \left. \pm\left(\begin{array}{rrrr}
0 & 0 & 0 & -1 \\
0 & 0 & 1 & 0 \\
0 & -1 & 0 & 0 \\
1 & 0 & 0 & 0
\end{array}\right)\right\}
\end{aligned}
$$

a group of order 8 .
For $\mathbb{Z}^{8}$, it is easiest to start by specifying the group. Let

$$
\gamma_{1}=\left(\begin{array}{rrrrrrrr}
0 & 1 & 0 & 0 & & & & \\
0 & 0 & 1 & 0 & & & & \\
0 & 0 & 0 & 1 & & & & \\
-1 & 0 & 0 & 0 & & & & \\
& & & & 0 & 1 & 0 & 0 \\
& & & & 0 & 0 & 1 & 0 \\
& & & & 0 & 0 & 0 & 1 \\
& & & & -1 & 0 & 0 & 0
\end{array}\right)
$$

and

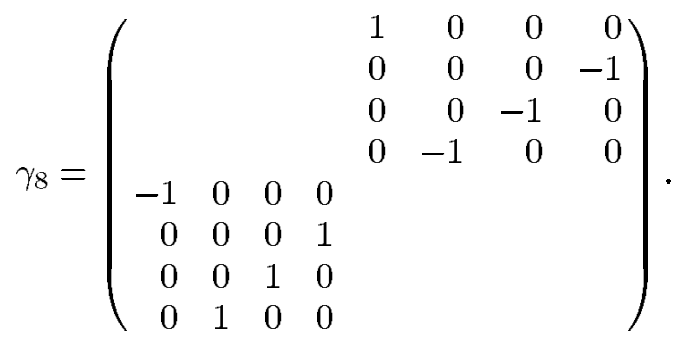

Then we take the group to be

$$
\Gamma=\left\{\gamma_{1}^{i}, \gamma_{8} \gamma_{1}^{i}, i=0,1,2, \ldots, 7\right\}
$$

a group of order 16. The generators for the lattices are $\boldsymbol{G}=I_{8}$ and $\boldsymbol{G}^{\prime}=\tilde{\boldsymbol{G}} \boldsymbol{G}$, where the $i$ th column of $\tilde{\boldsymbol{G}}$ is $\gamma_{i} v$, where $v=$ $(a 0 b 0 c 0 d 0)^{\operatorname{tr}}, a^{2}+b^{2}+c^{2}+d^{2}=N$, the index of $\Lambda^{\prime}$, and $i=0,1, \ldots, 7$.

Numerical computations for the lattices $\Lambda=\mathbb{Z}^{i}$ (standardized to have minimal length 1 ) for $i=1,2,4,8$, are presented in Fig. 14. These results have also been obtained for a uniformly distributed memoryless source. Since the two-channel distortions are identical for all the lattices considered in this figure, we have only plotted the excess distortion term

$$
(1 / N) \sum_{\lambda \in V_{0}(0)} d_{s}(\lambda)
$$

against the per-dimension reuse index $N^{1 / L}$.

\section{ASYMPTOTIC ANALYSIS}

We now derive upper and lower bounds on the distortion $\bar{d}_{s}$ as given by (15). As we have already seen in (28), the regularity of the labeling function and the high rate assumption lead to the following simplification for the expression in (15):

$$
\bar{d}_{s}=\bar{d}_{0}+\frac{1}{N} \sum_{\lambda \in V_{0}(0)} d_{s}(e)
$$

where $e$ is the label for the lattice point $\lambda$. Thus, in order to analyze $\bar{d}_{s}$ we only need to consider edges that label points in the discrete Voronoi set $V_{0}(0)$. Our analysis relies on a precise knowledge of the lengths of these edges. Suppose that $\lambda$ is labeled by the edge $e=\left(\lambda_{1}^{\prime}, \lambda_{2}^{\prime}\right)$, as in Fig. 7. Let $l(e)$ denote the length of the edge $e=\left\{\lambda_{1}^{\prime}, \lambda_{2}^{\prime}\right\}$, i.e., $l(e)=\left\|\lambda_{1}^{\prime}-\lambda_{2}^{\prime}\right\|$, and let 


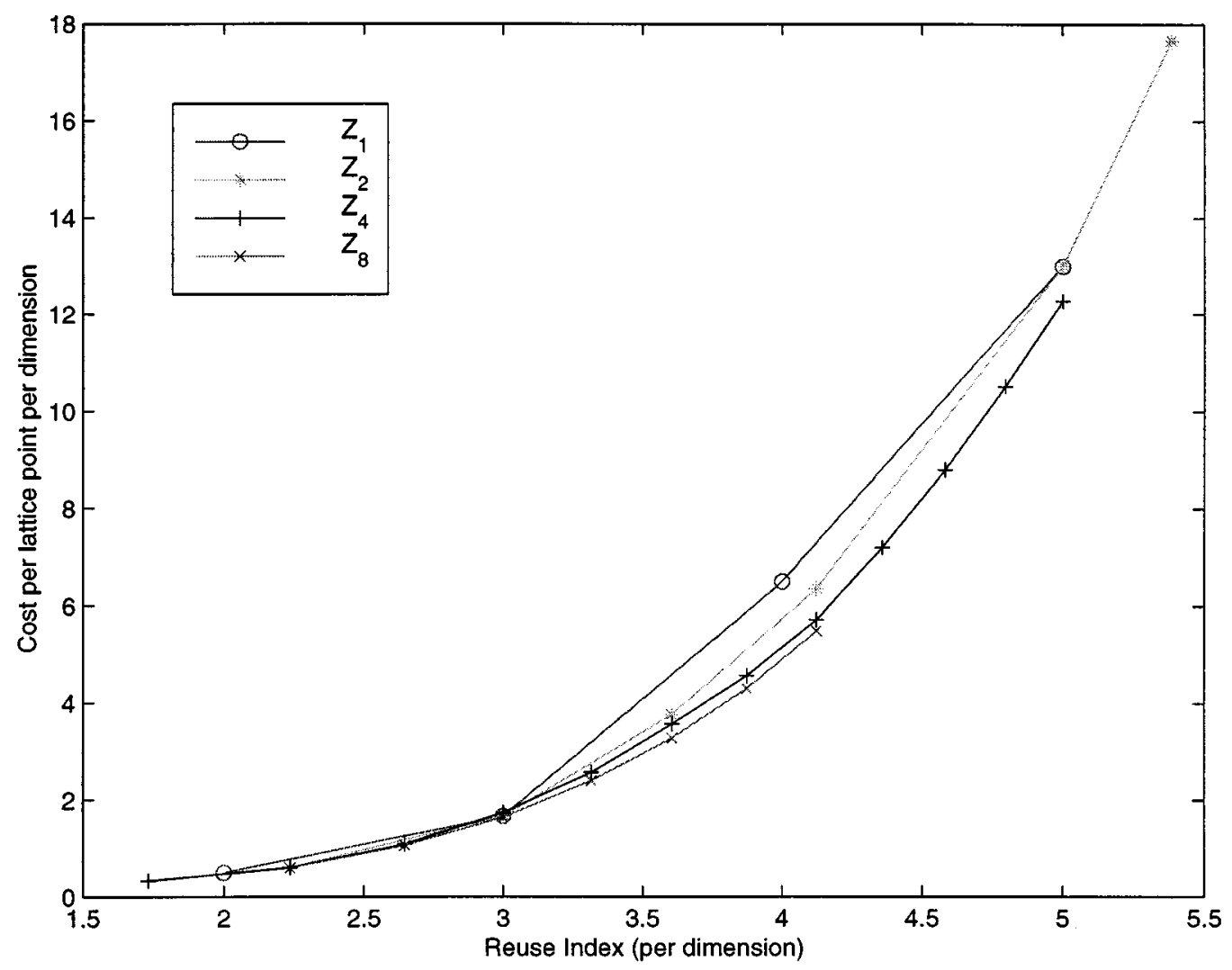

Fig. 14. A plot of $\bar{d}_{s}$ vs. the reuse index (per dimension) $N^{1 / L}$ for the lattices $\mathbb{Z}^{i}, i=1,2,4,8$.

$r$ be the distance of the point $\lambda$ from the midpoint $\left(\lambda_{1}^{\prime}+\lambda_{2}^{\prime}\right) / 2$. Then

$$
2 d_{s}(e)=\left\|\lambda-\lambda_{1}^{\prime}\right\|^{2}+\left\|\lambda-\lambda_{2}^{\prime}\right\|^{2}=(1 / 2) l(e)^{2}+2 r^{2} .
$$

A simple lower bound for $d_{s}(e)$ is obtained by setting $r$ to zero in the above equation. In order to obtain an upper bound, observe that the midpoint of a sublattice edge can always be made to lie in $V_{0}(0)$ by a suitable sublattice shift. An upper bound is then obtained (using the triangle inequality) by replacing $r$ with $r^{*}\left(\Lambda^{\prime}\right)=2 R\left(\Lambda^{\prime}\right)$, where $R\left(\Lambda^{\prime}\right)$ is the covering radius of the sublattice. We have

$$
(1 / 4) l^{2}(e) \leq d_{s}(e) \leq(1 / 4) l^{2}(e)+r^{*}\left(\Lambda^{\prime}\right)^{2}
$$

which by using (35) leads to the bounds

$$
\bar{d}_{0}+\frac{1}{4 N} \sum_{\lambda \in V_{0}(0)} l^{2}(e) \leq \bar{d}_{s} \leq \bar{d}_{0}+\frac{1}{4 N} \sum_{\lambda \in V_{0}(0)} l^{2}(e)+r^{*}\left(\Lambda^{\prime}\right)^{2} .
$$

\section{A. Asymptotic Performance}

In order to carry out a rate-distortion analysis, it is necessary to scale $\Lambda$ and $\Lambda^{\prime}$ by a real number $\beta$. We will use $\nu(\beta \Lambda)$ to denote the volume of a fundamental region for the scaled lattice $\beta \Lambda$. Clearly, $\nu(\beta \Lambda)=\beta^{L} \nu(\Lambda)$. For convenience we will write $\nu$ for $\nu(\Lambda)$. Upon rewriting (16) for the scaled lattice, we obtain

$$
\begin{aligned}
R_{0} & =h(p)-(1 / L) \log _{2}(\nu(\beta \Lambda)) \\
& =h(p)-(1 / L) \log _{2}\left(\beta^{L} \nu\right) .
\end{aligned}
$$

Similarly, an expression for $R$ is obtained by rewriting (17) in order to get

$$
\begin{aligned}
R & =h(p)-(1 / L) \log _{2}(N \nu(\beta \Lambda)) \\
& =h(p)-(1 / L) \log _{2}\left(N \beta^{L} \nu\right) .
\end{aligned}
$$

From (10), the two-channel distortion with the scaled lattice $\beta \Lambda$ is given by

$$
\bar{d}_{0}=G(\Lambda) \nu^{2 / L} \beta^{2}
$$

where we have used the fact that $G(\Lambda)=G(\beta \Lambda)$.

In terms of $R_{0}$, the two-channel distortion $\bar{d}_{0}$ is thus given by

$$
\bar{d}_{0}=G(\Lambda) 2^{2 h(p)} 2^{-2 R_{0}}
$$

Now let $N=2^{L(a R+1)}$. Then $R_{0}=R(1+a)+1$ and

$$
\bar{d}_{0}=\frac{G(\Lambda) 2^{2 h(p)}}{4} 2^{-2 R(1+a)} .
$$

For given $N$ and $R$, the correct scale factor is obtained by solving (41) for $\beta$

$$
\beta^{L}=\frac{2^{L h(p)} 2^{-L R(1+a)}}{2^{L} \nu} .
$$


Consider $\tilde{d}$ defined by

$$
\tilde{d}=\frac{1}{4 N} \sum_{\lambda \in V_{0}(0)} l^{2}(e) \beta^{2}
$$

which is the common term in the bounds for $\bar{d}_{s}$ given in (38). The quantity $\beta^{2}$ arises because we use the scaled lattices $\beta \Lambda$ and $\beta \Lambda^{\prime}$. It is understood that $e$ is the edge that labels $\lambda$. The edges in question in (45) (the edges in $\mathcal{E}_{d}(0)$ ) have been obtained by choosing the $N$ shortest edges in $\beta \Lambda^{\prime}$, with one endpoint at 0 and then shifting these edges so that the midpoint is as close to the origin as possible. Thus, each $l^{2}(e)$ is of the form $i N^{2 / L} / L$ for some $i \in \mathbb{Z}$. The term $N^{2 / L}$ is a scale factor that comes from the fact that $\left[\Lambda: \Lambda^{\prime}\right]=N$ and we normalize by $L$ because we are working with normalized square lengths. Let the largest value of $l^{2}(e)$ in (45) be equal to $K N^{2 / L} / L$ and let $B_{i}$ be the number of $l^{2}(e)$ 's that are equal to $i N^{2 / L} / L$. Then

$$
\tilde{d}=\frac{1}{4 N} \sum_{i=0}^{K} \frac{\beta^{2} i B_{i} N^{2 / L}}{L} .
$$

Our construction of the set $\mathcal{E}_{d}(0)$ implies that the $B_{i}$ can be obtained in terms of the coefficients of the theta series of the lattice $\Lambda$. To be specific, if $\Theta_{\Lambda}(z)=\sum_{i} A_{i} z^{i}$ is the theta series 3 for the lattice $\Lambda$, then we can assert that

$$
\begin{aligned}
B_{i} & =A_{i}, \quad 0 \leq i<K \\
B_{K} & \leq A_{K} .
\end{aligned}
$$

This fact will be used a little later.

Now substitute $\beta$ from (44) and use the fact that $2^{-2 a R}=$ $4 / N^{2 / L}$, in order to obtain

$$
\begin{aligned}
\tilde{d} & =\frac{2^{2 h(p)} 2^{-2 R(1-a)}}{\nu^{2 / L} N^{(1+2 / L)} L} \sum_{i=0}^{K} i B_{i} \\
& =\Xi \sum_{i=0}^{K} i B_{i}
\end{aligned}
$$

where we have defined

$$
\Xi=2^{2 h(p)} 2^{-2 R(1-a)} /\left(\nu^{2 / L} N^{(1+2 / L)} L\right) .
$$

The term $\sum_{i=0}^{K} i B_{i}$ can be bounded in terms of the $A_{i}$ 's by

$$
\sum_{i=0}^{K-1} i A_{i} \leq \sum_{i=0}^{K} i B_{i} \leq \sum_{i=0}^{K} i A_{i}
$$

Upon defining $S(m)=\sum_{i=0}^{m} A_{i}$ and using Abel's summation formula we obtain

$$
\sum_{i=0}^{m} i A_{i}=m S(m)-\sum_{n=0}^{m-1} S(n) .
$$

The term $S(n)$ is the number of lattice points in the first $n$ shells of the lattice $\Lambda$. This is roughly the ratio of the volume of $S_{L}\left(n^{1 / 2}\right)$, a sphere of radius $n^{1 / 2}$, to $\nu$, the volume of the Voronoi cell of $\Lambda$. To be specific, if $B_{L}$ denotes the volume of a sphere of unit radius in $\mathbb{R}^{L}$ then

$$
\lim _{n \rightarrow \infty} S(n) / n^{L / 2}=B_{L} / \nu \text {. }
$$

Thus $S(n)$ is given by

$$
S(n)=\frac{B_{L} n^{L / 2}}{\nu}(1+o(1))
$$

${ }^{3} A_{i}$ is the number of lattice points $\lambda$ with $L\|\lambda\|^{2}=i$. where $\lim _{n \rightarrow \infty} o(1)=0$. Using (51) in order to estimate $S(m)$, we obtain

$$
\begin{aligned}
\sum_{i=0}^{m} i A_{i}= & \left(\frac{m B_{L} m^{L / 2}}{\nu}-\sum_{n=0}^{m-1} \frac{B_{L} n^{L / 2}}{\nu}\right)(1+o(1)) \\
= & \frac{B_{L}}{\nu}\left(m^{L / 2+1}-\sum_{n=0}^{m-1} n^{L / 2}\right)(1+o(1)) \\
= & \frac{B_{L}}{\nu}\left(m^{(L / 2+1)}-\frac{m^{(L / 2+1)}}{(L / 2+1)}+o\left(m^{L / 2+1}\right)\right) \\
& \cdot(1+o(1)) \\
= & \frac{B_{L}}{\nu} \frac{L}{L+2} m^{(L / 2+1)}(1+o(1)) .
\end{aligned}
$$

On substituting (52) into (48) and observing from (51) that $N=$ $\left(B_{L} K^{L / 2} / \nu\right)(1+o(1))$ we obtain

$$
\tilde{d}=\frac{1}{B_{L}^{2 / L}(L+2)} 2^{2 h(p)} 2^{-2 R(1-a)}(1+o(1)) .
$$

But

$$
B_{L}=\frac{\pi^{L / 2}}{\Gamma(L / 2+1)}
$$

and $G\left(S_{L}\right)$, the normalized second moment of a sphere in $L$ dimensions, is given by

$$
G\left(S_{L}\right)=\frac{1}{(L+2) \pi} \Gamma(L / 2+1)^{2 / L} .
$$

Thus $B_{L}$ is given in terms of $G\left(S_{L}\right)$ by

$$
B_{L}^{2 / L}=\frac{1}{G\left(S_{L}\right)(L+2)}
$$

and from (53) it follows that

$$
\tilde{d}=G\left(S_{L}\right) 2^{2 h(p)} 2^{-2 R(1-a)}(1+o(1)) .
$$

The other terms in (38) are $\bar{d}_{0}$ and $r^{*}\left(\Lambda^{\prime}\right)^{2}$. The term $\bar{d}_{0}$ decays as $2^{-2 R(1+a)}$ and $r^{*}(\Lambda)^{2}$ decays like $\beta^{2} N^{2 / L}$, which in turn decays as $2^{-2 R}$. Thus

$$
\lim _{R \rightarrow \infty} \bar{d}_{s} 2^{2 R(1-a)}=\lim _{R \rightarrow \infty} \tilde{d} 2^{2 R(1-a)}
$$

and we have obtained our final result

$$
\lim _{R \rightarrow \infty} \bar{d}_{s} 2^{2 R(1-a)}=G\left(S_{L}\right) 2^{2 h(p)} .
$$

We end with a comparison with the multiple-description rate distortion bound (4), by letting $L \rightarrow \infty$ in (43) and (58). It is believed that as $L \rightarrow \infty, G(\Lambda) \rightarrow 1 / 2 \pi e$ and it is easily shown from (55) that $\lim _{L \rightarrow \infty} G\left(S_{L}\right)=1 / 2 \pi e$. Thus our constructions are optimal.

\section{SUMMARY AND CONCLUSION}

The problem of lattice vector quantizer design is addressed for the two-channel multiple description. The main problem in the design, a labeling problem, is solved. A systematic construction technique is developed which is suitable for general lattices. Specific constructions have been provided for $A_{2}$ and $\mathbb{Z}^{i}$, $i=1,2,4,8$. Finally, an asymptotic analysis reveals that performance arbitrarily close to the multiple-description rate distortion bound can be obtained. 
Open issues related to this work are detailed constructions for other lattices, extensions to the asymmetric case, and extensions to greater than two descriptions.

\section{ACKNOWLEDGMENT}

The authors wish to thank G. Nebe for suggesting the group of order 16 that we used for the lattice $\mathbb{Z}^{8}$. They also wish to thank the reviewers for their detailed and thoughtful comments and suggestions.

\section{REFERENCES}

[1] J.-P. Adoul and M. Barth, "Nearest neighbor algorithm for spherical codes from the Leech lattice," IEEE Trans. Inform. Theory, vol. 34, pp. 1188-1202, Sept. 1988.

[2] L. Auslander, Differential Geometry. New York: Harper and Row, 1967.

[3] R. Balan, I. Daubechies, and V. A. Vaishampayan, "Trading rate for distortion through varying the redundancy of a windowed Fourier frame in a multiple description compression," preprint, 1999.

[4] J.-C. Batllo and V. A. Vaishampayan, "Multiple-description transform codes with an application to packetized speech," in Proc. 1994 IEEE Int. Symp. Information Theory, Trondhiem, Norway, 1994.

[5] T. Berger, Rate Distortion Theory: A Mathematical Basis For Data Compression. Englewood Cliffs, NJ: Prentice-Hall, 1971.

[6] M. Bernstein, N. J. A. Sloane, and P. E. Wright, "On sublattices of the hexagonal lattice," Discr. Math., vol. 170, pp. 29-39, 1997.

[7] P. A. Chou, S. Mehrotra, and A. Wang, "Decoding of overcomplete expansions using projections onto convex sets," in Proc. 1999 Data Compression Conf., 1999, pp. 72-81.

[8] J. H. Conway, E. M. Rains, and N. J. A. Sloane, "On the existence of similar sublattices," Canad. J. Math., vol. 51, pp. 1300-1306, 1999.

[9] J. H. Conway and N. J. A. Sloane, "Voronoi regions of lattices, second moments of polytopes, and quantization," IEEE Trans. Inform. Theory, vol. IT-28, pp. 211-226, Mar. 1982.

[10] _ - "Fast quantizing and decoding algorithms for lattice quantizers and codes," IEEE Trans. Inform. Theory, vol. IT-28, pp. 227-232, Mar. 1982.

[11] — Sphere Packings, Lattices and Groups, 3rd ed. New York: Springer-Verlag, 1998

[12] A. A. El Gamal and T. M. Cover, "Achievable rates for multiple descriptions,” IEEE Trans. Inform. Theory, vol. IT-28, pp. 851-857, Nov. 1982.
[13] M. Fleming and M. Effros, "Generalized multiple description vector quantization," in Proc. 1999 Data Compression Conf., 1999, pp. 3-12.

[14] G. D. Forney, "Coset Codes-Part II," IEEE Trans. Inform. Theory, vol. 34, pp. 1152-1187, Sept. 1988

[15] A. Gersho, "Asymptotically optimal block quantization," IEEE Trans. Inform. Theory, vol. IT-25, pp. 373-380, July 1979.

[16] H. Gish and J. N. Pierce, "Asymptotically efficient quantizing," IEEE Trans. Inform. Theory, vol. IT-14, pp. 676-683, Sept. 1968.

[17] V. K. Goyal, J. Kovacevic, and M. Vetterli, "Quantized frame expansions as source-channel codes for erasure channels," in Proc. 1999 Data Compression Conf., 1999, pp. 326-335.

[18] R. M. Gray, Source Coding Theory. Norwell, MA: Kluwer, 1990.

[19] A. Ingle and V. A. Vaishampayan, "DPCM system design for diversity systems with applications to packetized speech," IEEE Trans. Speech and Audio Processing, vol. 1, pp. 48-58, Jan. 1995.

[20] H. Jafarkhani and V. Tarokh, "Multiple description trellis coded quantizers," IEEE Trans. Commun., vol. 47, pp. 799-803, June 1999

[21] D. G. Luenberger, Linear and Nonlinear Programming, 2nd ed. Reading, MA: Addison Wesley, 1984.

[22] A. E. Mohr, E. A. Riskin, and R. E. Ladner, "Graceful degradation over packet erasure channels through forward error correction," in Proc. 1999 Data Compression Conf., 1999, pp. 92-101.

[23] M. Orchard, Y. Wang, V. Vaishampayan, and A. Reibman, "Redundancy rate distortion analysis of multiple description coding using pairwise correlating transforms," in Proc. 1997 Int. Conf. Image Processing, 1997

[24] L. Ozarow, "On a source coding problem with two channels and three receivers,” Bell Syst. Tech. J., vol. 59, pp. 1909-1921, Dec. 1980.

[25] S. D. Servetto, V. A. Vaishampayan, and N. J. A. Sloane, "Multiple description lattice vector quantization," in Proc. 1999 Data Compression Conf., 1999, pp. 13-22.

[26] V. A. Vaishampayan, "Design of multiple description scalar quantizers," IEEE Trans. Inform. Theory, vol. 39, pp. 821-834, May 1993.

[27] V. Vaishampayan and J.-C. Batllo, "Asymptotic analysis of multiple description quantizers," IEEE Trans. Inform. Theory, vol. 44, pp. 278-284, Jan. 1998.

[28] V. Vaishampayan, J.-C. Batllo, and A. R. Calderbank, "On reducing granular distortion in multiple description quantization," in Proc. IEEE Int. Symp. Information Theory, Cambridge, MA, Aug. 1998, p. 98.

[29] V. Vaishampayan and A. A. Siddiqui, "Speech predictor design for diversity communication systems," in Proc. 1995 IEEE Speech Coding Workshop, 1995.

[30] A. Vardy and Y. Be'ery, "Maximum likelihood decoding of the Leech lattice," IEEE Trans. Inform. Theory, vol. 39, pp. 1435-1444, July 1993.

[31] P. Zador, "Asymptotic quantization error of continuous signals and the quantization dimension," IEEE Trans. Inform. Theory, vol. IT-28, pp. 139-149, Mar. 1982 